\title{
On the evolution of rapidly rotating massive white dwarfs towards supernovae or collapses
}

\author{
S.-C. Yoon ${ }^{\star}$ and N. Langer
}

\author{
Astronomical Institute, Utrecht University, Princetonplein 5, 3584 CC, Utrecht, The Netherlands \\ e-mail: scyoon@science.uva.nl; n.langer@astro.uu.nl
}

Received 15 December 2004 / Accepted 1 February 2005

\begin{abstract}
A recent study indicated that the inner cores of rapidly accreting $\left(\dot{M}>10^{-7} M_{\odot} \mathrm{yr}^{-1}\right)$ CO white dwarfs may rotate differentially, with a shear rate near the threshold value for the onset of the dynamical shear instability. Such differentially rotating white dwarfs have critical masses for thermonuclear explosion or electron-capture induced collapse that significantly exceed the canonical Chandrasekhar limit. Here, we construct two-dimensional differentially rotating white dwarf models with rotation laws resembling those of the one-dimensional models of the previous work. We derive analytic relations between the white dwarf mass, its angular momentum, and its rotational-, gravitational- and binding energy. We show that these relations are applicable for a wide range of angular velocity profiles, including solid body rotation. Taken at a central density of $2 \times$ $10^{9} \mathrm{~g} \mathrm{~cm}^{-3}$ they specify initial models for the thermonuclear explosion of rotating CO white dwarfs. At $\rho_{\mathrm{c}}=10^{10} \mathrm{~g} \mathrm{~cm}^{-3}$ and $4 \times 10^{9} \mathrm{~g} \mathrm{~cm}^{-3}$, they give criteria for the electron-capture induced collapse of rotating $\mathrm{CO}$ and $\mathrm{ONeMg}$ white dwarfs, respectively. We demonstrate that pre-explosion and pre-collapse conditions of both rigidly and differentially rotating white dwarfs are well established by the present work, which may facilitate future multi-dimensional simulations of type Ia supernova explosions and studies of the formation of millisecond pulsars and gamma-ray bursts from collapsing white dwarfs. Our results lead us to suggest various possible evolutionary scenarios for progenitors of type Ia supernovae, leading to a new paradigm of a variable mass of exploding white dwarfs, at values well above the classical Chandrasekhar mass. Based on our 2D-models, we argue that the supernova peak brightness is proportional to the white dwarf mass, which could explain various aspects of the diversity of type Ia supernovae, such as their variation in brightness, the dependence of their mean luminosity on the host galaxy type, and the weak correlation between ejecta velocity and peak brightness.
\end{abstract}

Key words. stars: rotation - stars: white dwarfs - stars: neutron - stars: supernovae: general - gamma rays: bursts

\section{Introduction}

Rotation, a universal phenomenon in celestial objects, is one of the most classical topics in stellar astrophysics (Tassoul 1978). Today, rotation is considered as an important ingredient in stellar evolution (Heger \& Langer 2000; Maeder \& Meynet 2000). Key aspects of massive star deaths - notably the production of millisecond pulsars, hypernovae and gamma-ray bursts require rapid rotation (e.g., Woosley 1993; Paczyński 1998; Hirschi et al. 2004; Heger et al. 2004; Petrovic et al. 2005b). In close binary systems, the effects of rotation become even more important due to the exchange of mass and angular momentum between the stellar components (Langer et al. 2004). For instance, the accreting stars in massive close binaries may be spun up to critical rotation, which significantly influences their evolution and final fate (Petrovic et al. 2005a).

It is hence surprising that for the last two decades, the effects of rotation on type Ia supernova (SN Ia) progenitors objects crucial for understanding chemical evolution as well as

* Present address: Astronomical Institute "Anton Pannekoek", University of Amsterdam, Kruislaan 403, 1098 SJ, Amsterdam, The Netherlands. the expansion history of the universe (e.g. Leibundgut 2001) have not been considered in detail. The currently favored Single Degenerate scenario for the evolution of SNe Ia progenitors assumes that the white dwarf accretes matter from a non-degenerate companion with high accretion rates $(\dot{M}=$ $\left.10^{-7} \ldots 10^{-6} M_{\odot}\right)$, to grow to the Chandrasekhar limit by steady nuclear shell burning (Hachisu et al. 1996; Li \& van den Heuvel 1997; Langer et al. 2000; Yoon \& Langer 2003; Han \& Podsiadlowski 2004). The white dwarf is supposed to accrete matter through a Keplerian disk, implying that the accreted matter carries large amounts of angular momentum (e.g. Langer et al. 2000). Here, as well as in alternative SN Ia progenitor scenarios, such as the sub-Chandrasekhar Mass scenario or the Double Degenerate scenario (see below), a spin-up of the accreting white dwarfs seems unavoidable. As an understanding of their diversity is essential for the use of SNe Ia to measure cosmological distances (e.g. Leibundgut 2001), the effects of rotation need to be carefully studied, since they could influence both the SNe Ia progenitor evolution and the thermonuclear explosion of the white dwarf. 
During the last two years, various systematic investigations of the effects of the white dwarf spin-up due to accretion in the context of SNe Ia progenitor evolution have emerged (Piersanti 2003a,b; Uenishi 2003; Yoon \& Langer 2004a,b; Yoon et al. 2004a; Saio \& Nomoto 2004). These results may demand surprising modifications of the standard picture of $\mathrm{SNe}$ Ia progenitors.

Yoon et al. (2004a) showed that the shell burning in rapidly accreting white dwarfs is stabilized by the accretion-induced spin-up, as the shell source is widened through rotationally induced chemical mixing and decompressed by the centrifugal support (cf. Yoon et al. 2004b). This result supports the key assumption of the Single Degenerate scenario that white dwarfs can efficiently grow in mass by shell burning, which has been challenged by the finding of generally unstable helium shell burning and subsequent mass loss in non-rotating white dwarfs (Cassisi et al. 1998; Kato \& Hachisu 2004).

In the sub-Chandrasekhar Mass scenario, the detonation of a thick helium layer triggers the explosion of a subChandrasekhar mass CO core (e.g. Woosley \& Weaver 1994). Here, Yoon \& Langer (2004b) showed that as the white dwarf is spun up by accretion at the moderate rates required to build up the degenerate helium layer, the latter is efficiently heated by the dissipation of rotational energy. Consequently, helium ignites much earlier and under less degenerate conditions than in the corresponding non-rotating case, which avoids a supernova explosion altogether.

Most intriguingly, a study by Yoon \& Langer (2004a; hereafter Paper I) indicated that accreting white dwarfs with $\dot{M} \gtrsim$ $10^{-7} M_{\odot} \mathrm{yr}^{-1}$ may rotate differentially throughout. They found that, although the strongly degenerate inner core is susceptible to the dynamical shear instability (DSI), other instabilities such as Eddington-Sweet circulations, the secular shear instability and the Goldreich, Schubert and Fricke instability are relevant for angular momentum redistribution at the required time scales $\left(\sim 10^{6} \mathrm{yr}\right)$ only in the non-degenerate outer layers. Accordingly, the shear rate in the inner core of the white dwarf corresponds to the shear rate near the threshold value for the onset of the DSI (Paper I). Since differentially rotating white dwarfs can be dynamically stable even up to $4.0 M_{\odot}$, the masses of exploding white dwarfs could be significantly larger than the canonical Chandrasekhar limit of $\sim 1.4 M_{\odot}$ (cf. Paper I). This may require us to supersede the long-standing paradigm of Chandrasekhar-mass white dwarfs as SN Ia progenitors with a new paradigm of SN Ia progenitors as white dwarfs with a variety of masses at super-Chandrasekhar mass values.

The possibility of super-Chandrasekhar mass white dwarfs is not new; in the 1960s, e.g. Ostriker \& Bodenheimer (1968) constructed differentially rotating $2 \mathrm{D}$ white dwarf models of up to $4.1 M_{\odot}$. However, the internal angular momentum distribution in these models had to be assumed and was chosen such that the spin rate decreases outward - conversely to the result of evolutionary considerations (Paper I). More recently, Uenishi et al. (2003) provided 2D white dwarf models, assuming a rotation law which introduces a discontinuity between the non-rotating inner core and the rigidly rotating spun-up envelope. However, due to this assumption, the maximum possible white dwarf mass became $1.47 M_{\odot}$, which strongly restricts the diversity of possible explosion masses. In the present paper, we construct 2D white dwarf models with rotation laws that mimic the 1D results of Paper I and thus follow from calculating the evolution of the internal white dwarf angular momentum profile throughout the accretion phase.

The remainder of this paper is organized as follows. In Sect. 2, we explain the numerical method and physical assumptions for constructing 2D white dwarf models. Basic model properties are described in Sect. 3, and, in the next section (Sect. 4), we present the quantitative criteria for thermonuclear explosion and electron-capture (EC) induced collapse of white dwarfs in terms of the white dwarf mass and total angular momentum. In Sect. 5, the 1D models of Paper I are compared to the 2D models of the present study. We discuss implications of our results for the evolution of accreting white dwarfs, and suggest new evolutionary scenarios for SNe Ia progenitors in Sect. 6. Possible consequences for the diversity of SN Ia are elaborated in Sect. 7, while implications of the EC induced collapse of differentially rotating white dwarfs are briefly discussed in Sect. 8. We summarize our main conclusions in Sect. 9.

\section{Constructing 2D models}

\subsection{Basic assumptions}

Rotating stars are called barotropic if the following two conditions are satisfied (Tassoul 1978):

$P=P(\rho)$ and $\frac{\partial \Omega}{\partial z}=0$

where $P$ is the pressure, $\rho$ the density $\Omega$ the angular velocity and $z$ the distance to the equatorial plane, respectively. Degenerate cores of accreting white dwarfs are thought to be barotropic on two accounts. First, the equation of state of highly electron-degenerate matter is given by $P=P(\rho)$. Second, perturbations which create $\frac{\partial \Omega}{\partial z} \neq 0$ will decay on a short time scale in degenerate matter, as discussed in Kippenhahn \& Möllenhof (1974) and Durisen (1977). Since the mass of the non-degenerate outer envelope in an accreting white dwarf is usually small (Paper I; Yoon et al. 2004a), the barotropic condition may be a good approximation for describing the whole white dwarf. For constructing axisymmetric 2D white dwarf models, we adopt cylindrical rotation as described below, and the equation of state of a completely degenerate electron gas, as in Ostriker \& Bodenheimer (1968):

$P=A\left[x\left(2 x^{2}-3\right)\left(x^{2}+1\right)^{1 / 2}+3 \sinh ^{-1} x\right] ; \rho=B x^{3}$,

where $A=6.01 \times 10^{22}$ dynes $\mathrm{cm}^{-2}$ and $B=9.82 \times$ $10^{5} \mu_{\mathrm{e}} \mathrm{g} \mathrm{cm}^{-3}$. Here we adopt $\mu_{\mathrm{e}}=2$ for all calculations.

\subsection{The rotation law}

According to Paper I, the rotation law in a white dwarf which accretes matter and angular momentum with accretion rates higher than $10^{-7} M_{\odot} / \mathrm{yr}$ is critically affected by the angular momentum transport due to the dynamical shear instability 
(DSI) in the inner core, and due to the secular shear instability, Eddington-Sweet circulations and the GSF instability in the outer layers. In particular, the shear rate in the inner core remains near the threshold value for the onset of the DSI $\left(:=\sigma_{\text {DSI,crit }}\right)$ during the accretion phase (see Paper I for more details). Another important feature of the rotation law is the presence of an absolute maximum in the angular velocity profile just above the shear-unstable core, which occurs due to the compression of the accreted layers with time (cf. also Durisen 1977 and see Fig. 9 in Paper I). From these constraints, we can construct a realistic rotation law as follows.

We define $s_{\mathrm{p}}$ as the position of the maximum angular velocity. As discussed in Paper I, above this position the buoyancy force is strong enough to assure stability against the DSI. In the white dwarf models in Paper I, this position is linked to layers with a density as low as several percent of the central density $\left(\rho_{\mathrm{c}}\right)$. Therefore, we determine $s_{\mathrm{p}}$ such that

$\rho\left(s=s_{\mathrm{p}}, z=0\right)=f_{\mathrm{p}} \rho_{\mathrm{c}}$.

In our models, we use $f_{\mathrm{p}}=0.1$ in most cases. Then, $\Omega(s)$ is determined in the range of $0 \leq s \leq s_{\mathrm{p}}$ from the fact that, in the inner core, the shear rate is given by

$\frac{\mathrm{d} \Omega(s)}{\mathrm{d} \ln s}=f_{\mathrm{sh}} \sigma_{\text {DSI,crit }}$.

Here, $s$ is the distance from the rotation axis in the cylindrical coordinate and the dimensionless parameter $f_{\mathrm{sh}}(\leq 1.0)$ describes the deviation of the shear rate from $\sigma_{\text {DSI,crit }}$. We use $f_{\text {sh }}=1.0$ except for some test cases. The threshold value of the shear rate for the onset of the DSI is given as $\sigma_{\text {DSI,crit }}=$ $\sqrt{N^{2} / R_{\mathrm{i}, \mathrm{c}}}$, where $N^{2}$ is the Brunt-Väisälä frequency and $R_{\mathrm{i}, \mathrm{c}}$ the critical Richardson number (see Paper I for more details). From this we get

$\Omega(s)=\Omega_{\mathrm{c}}+\int_{0}^{s} \frac{f_{\mathrm{sh}} \sigma_{\mathrm{DSI}, \mathrm{crit}}}{s^{\prime}} \mathrm{d} s^{\prime}$,

where $\Omega_{\mathrm{c}}$ is the angular velocity at the center. In order to ensure that $\mathrm{d} \Omega / \mathrm{d} s=0$ at $s=s_{\mathrm{p}}$, which is the case in 1D models of Paper I, we change the values of $\sigma_{\mathrm{DSI} \text {,crit }}$ around $s_{\mathrm{p}}$ such that

$\sigma_{\text {DSI,crit }}(s)=\left\{\begin{array}{ll}\sqrt{N^{2} / R_{\mathrm{i}, \mathrm{c}}} & \text { if } 0 \leq s \leq s_{\mathrm{b}} \\ \sqrt{N^{2} / R_{\mathrm{i}, \mathrm{c}}}\left(s-s_{\mathrm{p}}\right) /\left(s_{\mathrm{b}}-s_{\mathrm{p}}\right) & \text { if } s_{\mathrm{b}}<s \leq s_{\mathrm{p}}\end{array}\right.$,

where $s_{\mathrm{b}}$ is a certain location near to $s_{\mathrm{p}}$. In this study, we define $s_{\mathrm{b}}$ as the fifth neighboring grid point from $s_{\mathrm{p}}$ (while 149 grid points in $s$-direction are used). The calculation of $N^{2}$ is carried out by the use of the equation of state given by Blinnikov et al. (1996), using the equatorial values of $\rho$ and assuming $T=10^{8} \mathrm{~K}$ which is typical in the inner core of the accreting white dwarf models in Paper I. For the critical Richardson number, $R_{\mathrm{i}, \mathrm{c}}=0.25$ is used. The integration in Eq. (5) is carried out numerically by fitting $f_{\mathrm{sh}} \sigma_{\mathrm{DSI} \text {,crit }} / s$ with the Chebyshev polynomial (cf. Press et al. 1986).

At the surface the white dwarf equator is assumed to rotate at a certain fraction $\left(f_{\mathrm{K}}\right)$ of the Keplerian value, such that

$\Omega\left(R_{\mathrm{e}}\right)=f_{\mathrm{K}} \Omega_{\mathrm{K}}\left(R_{\mathrm{e}}\right)$, where $R_{\mathrm{e}}$ is the equatorial radius of the white dwarf, and $\Omega_{\mathrm{K}}\left(R_{\mathrm{e}}\right)=\sqrt{G M / R_{\mathrm{e}}^{3}}$. An accreting white dwarf may be expected to rotate near the critical velocity at its surface, since accretion through a Kelperian disk will carry angular momentum with the local Keplerian value. Therefore, we use $f_{\mathrm{K}}=0.95$ in most cases.

It remains to determine $\Omega(s)$ between $s_{\mathrm{p}}$ and $R_{\mathrm{e}}$. We impose an analytical form to $\Omega(s)$ in this region as follows:

$\Omega(s) / \Omega_{\mathrm{K}}(s)=\Omega\left(s_{\mathrm{p}}\right) / \Omega_{\mathrm{K}}\left(s_{\mathrm{P}}\right)+C\left(s-s_{\mathrm{p}}\right)^{a}$,

where $\Omega_{\mathrm{K}}(s)$ is the local Keplerian value. The constant $C$ can be determined with a given value of $a$ as

$C=\frac{f_{\mathrm{K}}-\Omega\left(s_{\mathrm{p}}\right) / \Omega_{\mathrm{K}}\left(s_{\mathrm{p}}\right)}{\left(R_{\mathrm{e}}-s_{\mathrm{p}}\right)^{a}}$.

The white dwarf structure is not significantly affected by the choice of the index $a$, because of the constraints imposed by $\Omega\left(s_{\mathrm{p}}\right)$ and $\Omega\left(R_{\mathrm{e}}\right)$ at each edge. Furthermore, the density in the outer region $\left(s>s_{\mathrm{p}}\right)$ is lower than the inner core, and the role of the rotation law of this region for the white dwarf structure is less important than that of the inner core. In the present study, $a=1.2$ is usually employed.

In summary, we determine the rotation law as follows. In the inner region $\left(0 \leq s \leq s_{\mathrm{p}}\right), \Omega(s)$ is determined by the dynamical shear instability (Eqs. (5) and (6)) for a given $\Omega_{\mathrm{c}}$. In the outer region ( $s_{\mathrm{p}} \leq s \leq R_{\mathrm{e}}$ ), $\Omega(s)$ is determined by Eq. (8) where $C$ is given by Eq. (9).

\subsection{SCF method}

For constructing 2D white dwarf models with the prescribed rotation law, we use the so-called self-consistent field (SCF) method which has been widely employed for modeling rotating stars (Ostriker \& Mark 1968; Hachisu 1986). This method can be most easily employed with barotropic stars, which satisfy the relation $\rho=\rho(\Psi)$, where the total potential $\Psi$ is the sum of the gravitational potential $\Phi$ and the centrifugal potential $\Theta$ :

$\Psi(\mu, r)=\Phi(\mu, r)+\Theta(s)$,

where

$\Phi(\mu, r)=G \int \frac{\rho\left(\boldsymbol{r}^{\prime}\right)}{\left|\boldsymbol{r}-\boldsymbol{r}^{\prime}\right|} \mathrm{d}^{3} \boldsymbol{r}^{\prime}$

and

$\Theta(s)=\int_{0}^{s} \Omega\left(s^{\prime}\right)^{2} s^{\prime} \mathrm{d} s^{\prime}$.

Here, $\mu(:=\cos \theta)$ and $r$ represent the spherical coordinates. The distance from the rotation axis $s$ is given by $s=r\left(1-\mu^{2}\right)^{1 / 2}$. In our study, the gravitational potential $\Phi$ is calculated using Simpson's formula following Hachisu (1986). For the centrifugal potential $\Theta$, the integration in Eq. (12) is carried out by approximating the integrand by Chebyshev polynomials.

The SCF method then proceeds as follows. Once we have $\Psi$ from a given trial density distribution $\rho(\mu, r)$ and a given angular velocity profile, a better value for the density $\rho$ can be given analytically as a function of $\Psi$ (see Ostriker \& Bodenheimer 1968). This new density distribution in turn gives a new value 
of $\Psi$. This procedure is iterated until a good convergence level is achieved. In the present study, the iteration continues until the following conditions are satisfied:

$$
\left|\frac{\Psi^{\text {new }}-\Psi^{\text {old }}}{\Psi^{\text {new }}}\right|_{\max }<5 \times 10^{-5}, \text { and }\left|\frac{C-\langle C\rangle}{\langle C\rangle}\right|_{\max }<5 \times 10^{-5}
$$

where $C$, which should be constant in ideal equilibrium structures, is defined as

$C=\int \rho^{-1} \mathrm{~d} P-\Phi-\Theta$,

and $\langle C\rangle$ denotes its mean value. One additional condition is imposed by the virial relation such that

$$
\frac{|2 T-W+3 \Pi|}{W}<10^{-3},
$$

where $T$ and $W$ denote rotational- and gravitational energy, respectively, and $\Pi$ the volume integral of the pressure. Note that here we define gravitational energy as a positive value for convenience. Hereafter, we will refer to the above explained procedure as SCF iteration.

\subsection{Further details}

We use the maximum density $\left(\rho_{\max }\right)$, the gravitational constant $(G)$ and the equatorial radius at the surface of the white dwarf $\left(R_{\mathrm{e}}\right)$ for the basis of dimensional variables for numerical calculations, as in Hachisu (1986). With this choice, the physical variables can be expressed in a dimensionless form such that $\hat{\rho}=\rho / \rho_{\max }, \hat{s}=s / R_{\mathrm{e}}, \hat{j}=j / \sqrt{G \rho_{\max }} R_{\mathrm{e}}^{2}, \hat{\Omega}=\Omega / \sqrt{G \rho_{\max }}$, $\hat{\Psi}=\Psi / G R_{\mathrm{e}}^{2} \rho_{\max }$, and so on. As pointed out by Hachisu (1986), using a fixed value of $\rho_{\max }$ instead of the total mass $M$ makes the numerical convergence faster, especially because the equatorial radius $R_{\mathrm{e}}$ can be given analytically as

$R_{\mathrm{e}}=\left\{\frac{8 A}{B} \frac{\left[1+\left(\rho_{\max }\right)^{2 / 3}\right]^{1 / 2}-1}{G \rho_{\max }\left(\hat{\Psi}_{\max }-\hat{\Psi}_{\mathrm{s}}\right)}\right\}^{1 / 2}$,

where $\hat{\Psi}_{\mathrm{s}}$ indicates the boundary value.

Hachisu also chose the axis ratio as an initially fixed parameter for determining the equilibrium structure. This approach is possible only when the rotation law is given analytically, and cannot be applied for the rotation law defined in Sect. 2.2. Instead, we choose the central angular velocity $\Omega_{\mathrm{c}}$ as an initial parameter. Therefore, in our models, initially fixed maximum density $\rho_{\max }$ and central angular velocity $\Omega_{\mathrm{c}}$ determine the equilibrium structure of the white dwarf, together with given values of $f_{\mathrm{p}}$ (Eq. (3)), $f_{\mathrm{sh}}$ (Eq. (4)), $f_{\mathrm{K}}$ (Eq. (7)) and $a$ (Eq. (9)). More specifically, at a given $\rho_{\max }$, the total angular momentum $J$ and the white dwarf mass $M$ increase with increasing $\Omega_{\mathrm{c}}$.

It is found that, when the centrifugal potential $\Theta$ is calculated directly from $\Omega(s)$ given by Eqs. (5) and (8), models do not converge well if the ratio of the rotational energy to the gravitational energy $(T / W)$ becomes rather high $(\gtrsim 0.07)$. This is due to the complexity of the prescribed rotation law. Usually, our numerical code works very efficiently even when $T / W>0.2$ if the rotation law is a well defined function of position as in the case of $v$-constant rotation or $j$-constant rotation (see Eqs. (17) and (18) below). We thus pre-determine the specific angular momentum $\hat{j}(s)$ from a trial density distribution before starting the SCF iteration such that $\hat{j}(\hat{s})=\hat{\Omega}(\hat{s}) \hat{s}^{2}$, where $\hat{\Omega}$ is obtained from Eqs. (5) and (8). We then introduce a new rotation law $\hat{\omega}(\hat{s})$ such that $\hat{\omega}(\hat{s})=\hat{j}(\hat{s}) / \hat{s}^{2}$. Although $\hat{\Omega}$ and $\hat{\omega}$ are identical at the beginning of the SCF iteration, the two deviate from each other during the SCF iteration, since $\hat{\Omega}$ depends on both density and position (see Eqs. (5) and (6)) while $\hat{\omega}$, given by the pre-determined $\hat{j}(\hat{s})$, depends only on position. Once the SCF integration is completed, we calculate $\hat{\Omega}$ again by the use of Eqs. (5) and (8) from the converged model, and determine a new $\hat{j}(\hat{s})$. Then, the SCF iteration is carried out again with the new $\hat{j}(\hat{s})$. This procedure is repeated until $\hat{\Omega}$ and $\hat{\omega}$ converge, within an accuracy of $3 \%$.

\subsection{Model sequences}

Hereafter, we will refer to the rotation law given by Eqs. (5) and (8) as accreting white dwarf rotation (AWD rotation). For comparison, we employ rigid, $v$-constant and $j$-constant rotation laws, which were also considered by Hachisu (1986).

Model sequences which are constructed in the present study with different parameters and different rotation laws are summarized as follows (see also Tables 1 and 2, below):

1. AWD rotation: $\Omega$ given by Eqs. (5) and (8)

- sequence $\mathrm{AA}: f_{\mathrm{sh}}=1.0, f_{\mathrm{K}}=0.95, f_{\mathrm{p}}=0.10, a=1.2$;

- sequence $\mathrm{AB}: f_{\mathrm{sh}}=1.0, f_{\mathrm{K}}=0.95, f_{\mathrm{p}}=0.05, a=1.2$;

- sequence $\mathrm{AC}: f_{\mathrm{sh}}=1.0, f_{\mathrm{K}}=0.95, f_{\mathrm{p}}=0.10, a=1.0$;

- sequence $\mathrm{AD}: f_{\mathrm{sh}}=1.0, f_{\mathrm{K}}=0.95, f_{\mathrm{p}}=0.20, a=1.2$;

- sequence $\mathrm{AE}: f_{\mathrm{sh}}=0.5, f_{\mathrm{K}}=0.50, f_{\mathrm{p}}=0.10, a=1.2$;

- sequence AF: $f_{\mathrm{sh}}=0.1, f_{\mathrm{K}}=0.95, f_{\mathrm{p}}=0.10, a=1.2$;

- sequence AG: $f_{\mathrm{sh}}=-1.0, f_{\mathrm{K}}=0.6, f_{\mathrm{p}}=0.01, a=0.9$;

2. rigid rotation (sequence $\mathrm{R}$ ): $\Omega=$ constant

3. $j$-constant rotation (sequence $\mathrm{J}$ ):

$$
\Omega=j_{0} /\left(0.01 R_{\mathrm{e}}^{2}+s^{2}\right) .
$$

4. $v$-constant rotation (sequence $\mathrm{V}$ ):

$$
\Omega=v_{0} /\left(0.01 R_{\mathrm{e}}^{2}+s^{2}\right)^{1 / 2} .
$$

Here, sequences $\mathrm{AA}, \mathrm{AB}$ and $\mathrm{AC}$ are intended to describe the properties of $1 \mathrm{D}$ models in Paper I. Sequences AD, AE and AF are to consider uncertainties due to changes of the given parameters (see below). Sequence AG is to investigate the white dwarf structure when the inner core rotates at the shear rate of $-\sigma_{\mathrm{DSI}, \text { crit }}$.

We consider model sequences at 7 different values of $\rho_{\max }$ : 1.0, 2.0, 5.0, 8.0, 20, 40.0, and $100 \times 10^{8} \mathrm{~g} \mathrm{~cm}^{-3}$. Each sequence is given a subindex ( $\mathrm{a}, \mathrm{b}, \mathrm{c}, \mathrm{d}, \mathrm{e}, \mathrm{f}$ and $\mathrm{g}$ ), in order to indicate the corresponding $\rho_{\max }$. For instance, AAa denotes the sequence with $f_{\mathrm{sh}}=1.0, f_{\mathrm{K}}=0.95, f_{\mathrm{p}}=0.10, a=1.2$, and with $\rho_{\max }=1.0 \times 10^{8} \mathrm{~g} \mathrm{~cm}^{-3}$, etc. Note that in all models with an AWD- or rigid rotation law the maximum density $\rho_{\max }$ corresponds to the central density $\rho_{\mathrm{c}}$. However, they may differ from each other for $v$-constant and $j$-constant rotation laws when the ratio of the rotational energy to the gravitational energy $(T / W)$ becomes sufficiently high. Within each sequence, 
Table 1. Properties of selected models in sequence AA. The columns has the following meaning. $M$ : white dwarf mass, $J$ : total angular momentum, $\Omega_{\mathrm{c}}$ : central angular velocity, $\Omega_{\mathrm{m}}$ : moment-of-inertia-weighted mean of angular velocity, $W$ : gravitational energy, $T:$ rotational energy, $U$ : internal energy, $T / W$ : ratio of the rotational energy to the gravitational energy, $R_{\mathrm{e}}$ : equatorial radius, $R_{\mathrm{p}} / R_{\mathrm{e}}$ : ratio of the polar radius to the equatorial radius.

\begin{tabular}{|c|c|c|c|c|c|c|c|c|c|c|}
\hline No. & $\begin{array}{c}M \\
{\left[M_{\odot}\right]}\end{array}$ & $\begin{array}{c}J \\
{\left[10^{50} \mathrm{erg} \mathrm{s}\right]}\end{array}$ & $\begin{array}{c}\Omega_{\mathrm{c}} \\
{[\mathrm{rad} / \mathrm{s}]}\end{array}$ & $\begin{array}{c}\Omega_{\mathrm{m}} \\
{[\mathrm{rad} / \mathrm{s}]}\end{array}$ & $\begin{array}{c}W \\
{\left[10^{50} \mathrm{erg}\right]}\end{array}$ & $\begin{array}{c}T \\
{\left[10^{50} \mathrm{erg}\right]}\end{array}$ & $\begin{array}{c}U \\
{\left[10^{50} \mathrm{erg}\right]}\end{array}$ & $\bar{T} T / W$ & $\begin{array}{c}R_{\mathrm{e}} \\
{\left[0.01 R_{\odot}\right]}\end{array}$ & $\bar{R} R_{\mathrm{p}} / R_{\mathrm{c}}$ \\
\hline \multicolumn{11}{|c|}{ Sequence AA: $f_{\mathrm{sh}}=1.0 ; f_{\mathrm{K}}=0.95 ; f_{\mathrm{p}}=0.1 ; a=1.2$} \\
\hline \multicolumn{11}{|c|}{$\rho_{\mathrm{c}}=1 \times 10^{8} \mathrm{~g} \mathrm{~cm}^{-3}$} \\
\hline AAa8 & 1.301 & 0.875 & 0.181 & 0.881 & 9.848 & 0.393 & 6.246 & 0.040 & 0.959 & 0.609 \\
\hline AAa13 & 1.351 & 1.095 & 0.310 & 0.964 & 10.351 & 0.536 & 6.386 & 0.052 & 1.026 & 0.558 \\
\hline AAa16 & 1.391 & 1.267 & 0.387 & 1.008 & 10.746 & 0.650 & 6.493 & 0.061 & 1.077 & 0.529 \\
\hline AAa 18 & 1.420 & 1.398 & 0.439 & 1.031 & 11.037 & 0.736 & 6.569 & 0.067 & 1.117 & 0.500 \\
\hline AAa20 & 1.455 & 1.561 & 0.491 & 1.051 & 11.380 & 0.841 & 6.654 & 0.074 & 1.167 & 0.478 \\
\hline AAa22 & 1.493 & 1.730 & 0.542 & 1.070 & 11.765 & 0.953 & 6.758 & 0.081 & 1.211 & 0.457 \\
\hline AAa23 & 1.530 & 1.911 & 0.568 & 1.075 & 12.120 & 1.064 & 6.841 & 0.088 & 1.264 & 0.428 \\
\hline AAa24 & 1.557 & 2.043 & 0.594 & 1.081 & 12.396 & 1.148 & 6.910 & 0.093 & 1.294 & 0.420 \\
\hline AAa 25 & 1.584 & 2.171 & 0.620 & 1.086 & 12.668 & 1.231 & 6.980 & 0.097 & 1.322 & 0.406 \\
\hline AAa26 & 1.619 & 2.355 & 0.646 & 1.083 & 13.003 & 1.339 & 7.055 & 0.103 & 1.377 & 0.391 \\
\hline AAa 27 & 1.656 & 2.540 & 0.671 & 1.085 & 13.384 & 1.454 & 7.150 & 0.109 & 1.408 & 0.377 \\
\hline AAa28 & 1.697 & 2.770 & 0.697 & 1.072 & 13.755 & 1.578 & 7.224 & 0.115 & 1.476 & 0.355 \\
\hline AAa29 & 1.756 & 3.097 & 0.749 & 1.066 & 14.358 & 1.771 & 7.365 & 0.123 & 1.522 & 0.341 \\
\hline AAa30 & 1.804 & 3.369 & 0.775 & 1.056 & 14.825 & 1.922 & 7.469 & 0.130 & 1.574 & 0.326 \\
\hline AAa31 & 1.869 & 3.736 & 0.788 & 1.051 & 15.512 & 2.137 & 7.630 & 0.138 & 1.612 & 0.319 \\
\hline \multicolumn{11}{|c|}{$\rho_{\mathrm{c}}=2 \times 10^{9} \mathrm{~g} \mathrm{~cm}^{-3}$} \\
\hline AAe2 & 1.432 & 0.361 & 0.115 & 2.280 & 30.699 & 0.426 & 24.812 & 0.014 & 0.414 & 0.696 \\
\hline AAe6 & 1.454 & 0.438 & 0.577 & 2.618 & 31.295 & 0.584 & 25.032 & 0.019 & 0.429 & 0.667 \\
\hline AAe8 & 1.466 & 0.479 & 0.808 & 2.776 & 31.638 & 0.674 & 25.161 & 0.021 & 0.437 & 0.652 \\
\hline AAe10 & 1.481 & 0.529 & 1.039 & 2.949 & 32.073 & 0.788 & 25.322 & 0.025 & 0.448 & 0.630 \\
\hline AAe12 & 1.496 & 0.577 & 1.270 & 3.096 & 32.501 & 0.903 & 25.478 & 0.028 & 0.457 & 0.616 \\
\hline AAe14 & 1.513 & 0.628 & 1.501 & 3.237 & 32.980 & 1.029 & 25.657 & 0.031 & 0.467 & 0.594 \\
\hline AAe18 & 1.553 & 0.749 & 1.963 & 3.507 & 34.143 & 1.336 & 26.087 & 0.039 & 0.492 & 0.558 \\
\hline AAe20 & 1.579 & 0.825 & 2.194 & 3.640 & 34.874 & 1.533 & 26.353 & 0.044 & 0.507 & 0.529 \\
\hline AAe22 & 1.605 & 0.904 & 2.425 & 3.754 & 35.652 & 1.743 & 26.635 & 0.049 & 0.523 & 0.507 \\
\hline AAe24 & 1.639 & 1.004 & 2.656 & 3.863 & 36.620 & 2.006 & 26.978 & 0.055 & 0.543 & 0.486 \\
\hline AAe26 & 1.677 & 1.118 & 2.887 & 3.952 & 37.695 & 2.303 & 27.352 & 0.061 & 0.565 & 0.464 \\
\hline AAe 28 & 1.721 & 1.255 & 3.118 & 4.023 & 38.932 & 2.657 & 27.769 & 0.068 & 0.592 & 0.435 \\
\hline AAe 30 & 1.759 & 1.373 & 3.349 & 4.069 & 40.027 & 2.964 & 28.142 & 0.074 & 0.614 & 0.413 \\
\hline AAe 32 & 1.841 & 1.633 & 3.580 & 4.109 & 42.342 & 3.622 & 28.908 & 0.086 & 0.659 & 0.377 \\
\hline AAe34 & 1.898 & 1.831 & 3.811 & 4.100 & 43.950 & 4.102 & 29.417 & 0.093 & 0.692 & 0.355 \\
\hline AAe36 & 1.952 & 2.016 & 4.042 & 4.082 & 45.460 & 4.546 & 29.898 & 0.100 & 0.723 & 0.333 \\
\hline AAe37 & 2.029 & 2.300 & 4.158 & 4.021 & 47.602 & 5.189 & 30.539 & 0.109 & 0.761 & 0.312 \\
\hline \multicolumn{11}{|c|}{$\rho_{\mathrm{c}}=4 \times 10^{9} \mathrm{~g} \mathrm{~cm}^{-3}$} \\
\hline AAf3 & 1.448 & 0.313 & 0.327 & 3.047 & 39.226 & 0.492 & 32.819 & 0.013 & 0.344 & 0.696 \\
\hline AAf5 & 1.457 & 0.346 & 0.653 & 3.283 & 39.560 & 0.579 & 32.949 & 0.015 & 0.350 & 0.681 \\
\hline AAf7 & 1.469 & 0.382 & 0.980 & 3.528 & 39.961 & 0.684 & 33.104 & 0.017 & 0.357 & 0.667 \\
\hline AAf9 & 1.480 & 0.419 & 1.307 & 3.753 & 40.387 & 0.795 & 33.272 & 0.020 & 0.363 & 0.645 \\
\hline AAf11 & 1.494 & 0.458 & 1.633 & 3.971 & 40.865 & 0.918 & 33.460 & 0.022 & 0.371 & 0.630 \\
\hline AAf13 & 1.508 & 0.501 & 1.960 & 4.181 & 41.391 & 1.057 & 33.663 & 0.026 & 0.378 & 0.616 \\
\hline AAf15 & 1.524 & 0.544 & 2.287 & 4.376 & 41.957 & 1.203 & 33.886 & 0.029 & 0.386 & 0.594 \\
\hline AAf17 & 1.543 & 0.596 & 2.613 & 4.583 & 42.648 & 1.385 & 34.156 & 0.032 & 0.396 & 0.572 \\
\hline AAf19 & 1.563 & 0.652 & 2.940 & 4.763 & 43.369 & 1.579 & 34.427 & 0.036 & 0.407 & 0.558 \\
\hline AAf21 & 1.585 & 0.710 & 3.267 & 4.934 & 44.171 & 1.791 & 34.736 & 0.041 & 0.418 & 0.536 \\
\hline AAf 23 & 1.607 & 0.767 & 3.593 & 5.073 & 44.956 & 1.997 & 35.038 & 0.044 & 0.428 & 0.514 \\
\hline AAf 25 & 1.637 & 0.846 & 3.920 & 5.232 & 46.047 & 2.289 & 35.449 & 0.050 & 0.443 & 0.493 \\
\hline AAf27 & 1.669 & 0.932 & 4.247 & 5.369 & 47.207 & 2.607 & 35.881 & 0.055 & 0.459 & 0.471 \\
\hline AAf 29 & 1.706 & 1.032 & 4.574 & 5.485 & 48.542 & 2.972 & 36.372 & 0.061 & 0.477 & 0.449 \\
\hline AAf31 & 1.750 & 1.155 & 4.900 & 5.568 & 50.128 & 3.413 & 36.934 & 0.068 & 0.500 & 0.420 \\
\hline AAf33 & 1.792 & 1.271 & 5.227 & 5.629 & 51.646 & 3.834 & 37.480 & 0.074 & 0.519 & 0.399 \\
\hline AAf35 & 1.880 & 1.527 & 5.554 & 5.672 & 54.784 & 4.736 & 38.566 & 0.086 & 0.562 & 0.362 \\
\hline AAf37 & 1.989 & 1.851 & 5.880 & 5.709 & 58.928 & 5.902 & 40.027 & 0.100 & 0.597 & 0.333 \\
\hline
\end{tabular}


Table 2. Same as in Table 1 but with sequences $\mathrm{ABe}, \mathrm{ACe}, \mathrm{ADe}, \mathrm{AEe}$ and AFe.

\begin{tabular}{|c|c|c|c|c|c|c|c|c|c|c|}
\hline No. & $\begin{array}{c}M \\
{\left[M_{\odot}\right]}\end{array}$ & $\begin{array}{c}J \\
{\left[10^{50} \mathrm{erg} \mathrm{s}\right]}\end{array}$ & $\begin{array}{c}\Omega_{\mathrm{c}} \\
{[\mathrm{rad} / \mathrm{s}]}\end{array}$ & $\begin{array}{c}\Omega_{\mathrm{m}} \\
{[\mathrm{rad} / \mathrm{s}]}\end{array}$ & $\begin{array}{c}W \\
{\left[10^{50} \mathrm{erg}\right]}\end{array}$ & $\begin{array}{c}T \\
{\left[10^{50} \mathrm{erg}\right]}\end{array}$ & $\begin{array}{c}U \\
{\left[10^{50} \mathrm{erg}\right]}\end{array}$ & $T / W$ & $\begin{array}{c}R_{\mathrm{e}} \\
{\left[0.01 R_{\odot}\right]}\end{array}$ & $R_{\mathrm{p}} / R_{\mathrm{e}}$ \\
\hline \multicolumn{11}{|c|}{ Sequence ABe: $f_{\mathrm{sh}}=1.0 ; f_{\mathrm{K}}=0.95 ; f_{\mathrm{p}}=0.05 ; a=1.2 ; \rho_{\mathrm{c}}=2 \times 10^{9} \mathrm{~g} \mathrm{~cm}^{-3}$} \\
\hline $\mathrm{ABe} 2$ & 1.467 & 0.483 & 0.635 & 2.766 & 31.615 & 0.682 & 25.124 & 0.022 & 0.442 & 0.645 \\
\hline $\mathrm{ABe} 10$ & 1.502 & 0.597 & 1.097 & 3.105 & 32.556 & 0.942 & 25.448 & 0.029 & 0.471 & 0.594 \\
\hline $\mathrm{ABe} 19$ & 1.554 & 0.763 & 1.617 & 3.437 & 33.955 & 1.337 & 25.920 & 0.039 & 0.515 & 0.536 \\
\hline $\mathrm{ABe} 23$ & 1.580 & 0.842 & 1.848 & 3.552 & 34.656 & 1.531 & 26.159 & 0.044 & 0.534 & 0.507 \\
\hline $\mathrm{ABe} 33$ & 1.685 & 1.175 & 2.425 & 3.780 & 37.397 & 2.325 & 27.040 & 0.062 & 0.617 & 0.428 \\
\hline ABe37 & 1.757 & 1.412 & 2.656 & 3.821 & 39.262 & 2.876 & 27.622 & 0.073 & 0.670 & 0.384 \\
\hline $\mathrm{ABe} 42$ & 1.857 & 1.775 & 2.945 & 3.719 & 41.633 & 3.624 & 28.280 & 0.087 & 0.758 & 0.333 \\
\hline $\mathrm{ABe} 48$ & 2.034 & 2.486 & 3.407 & 3.438 & 45.757 & 4.945 & 29.375 & 0.108 & 0.893 & 0.275 \\
\hline \multicolumn{11}{|c|}{ Sequence ACe: $f_{\mathrm{sh}}=1.0 ; f_{\mathrm{K}}=0.95 ; f_{\mathrm{p}}=0.1 ; a=1.0 ; \rho_{\mathrm{c}}=2 \times 10^{9} \mathrm{~g} \mathrm{~cm}^{-3}$} \\
\hline ACe5 & 1.457 & 0.450 & 0.462 & 2.631 & 31.321 & 0.607 & 25.011 & 0.019 & 0.451 & 0.638 \\
\hline $\mathrm{ACe} 13$ & 1.516 & 0.642 & 1.386 & 3.226 & 32.973 & 1.048 & 25.612 & 0.032 & 0.492 & 0.565 \\
\hline ACe19 & 1.579 & 0.833 & 2.079 & 3.596 & 34.765 & 1.528 & 26.267 & 0.044 & 0.533 & 0.507 \\
\hline $\mathrm{ACe} 25$ & 1.678 & 1.133 & 2.772 & 3.894 & 37.553 & 2.298 & 27.243 & 0.061 & 0.597 & 0.435 \\
\hline $\mathrm{ACe} 29$ & 1.753 & 1.366 & 3.234 & 3.997 & 39.697 & 2.895 & 27.981 & 0.073 & 0.641 & 0.399 \\
\hline ACe32 & 1.849 & 1.681 & 3.580 & 4.028 & 42.375 & 3.671 & 28.858 & 0.087 & 0.700 & 0.355 \\
\hline $\mathrm{ACe} 35$ & 1.969 & 2.102 & 3.927 & 3.973 & 45.693 & 4.654 & 29.893 & 0.102 & 0.771 & 0.312 \\
\hline \multicolumn{11}{|c|}{ Sequence ADe: $f_{\mathrm{sh}}=0.1 ; f_{\mathrm{K}}=0.95 ; f_{\mathrm{p}}=0.20 ; a=1.2 ; \rho_{\mathrm{c}}=2 \times 10^{9} \mathrm{~g} \mathrm{~cm}^{-3}$} \\
\hline ADe2 & 1.447 & 0.418 & 0.577 & 2.539 & 31.137 & 0.538 & 24.982 & 0.017 & 0.427 & 0.674 \\
\hline ADe6 & 1.597 & 0.868 & 2.887 & 3.783 & 35.646 & 1.685 & 26.746 & 0.047 & 0.502 & 0.529 \\
\hline ADe7 & 1.664 & 1.060 & 3.465 & 4.041 & 37.754 & 2.230 & 27.560 & 0.059 & 0.534 & 0.486 \\
\hline ADe8 & 1.740 & 1.276 & 4.042 & 4.221 & 40.100 & 2.849 & 28.438 & 0.071 & 0.565 & 0.442 \\
\hline ADe9 & 1.869 & 1.667 & 4.620 & 4.389 & 44.189 & 3.964 & 29.923 & 0.090 & 0.622 & 0.384 \\
\hline ADe10 & 2.044 & 2.233 & 5.197 & 4.464 & 49.892 & 5.547 & 31.919 & 0.111 & 0.688 & 0.333 \\
\hline \multicolumn{11}{|c|}{ Sequence AEe: $f_{\mathrm{sh}}=0.5 ; f_{\mathrm{K}}=0.5 ; f_{\mathrm{p}}=0.10 ; a=1.2 ; \rho_{\mathrm{c}}=2 \times 10^{9} \mathrm{~g} \mathrm{~cm}^{-3}$} \\
\hline AEe1 & 1.391 & 0.170 & 0.000 & 1.224 & 29.624 & 0.114 & 24.467 & 0.004 & 0.320 & 0.920 \\
\hline AEe2 & 1.422 & 0.319 & 1.155 & 2.132 & 30.530 & 0.342 & 24.822 & 0.011 & 0.331 & 0.870 \\
\hline AEe3 & 1.480 & 0.515 & 2.310 & 3.000 & 32.292 & 0.784 & 25.523 & 0.024 & 0.350 & 0.797 \\
\hline $\mathrm{AEe} 4$ & 1.578 & 0.792 & 3.465 & 3.744 & 35.252 & 1.538 & 26.673 & 0.044 & 0.377 & 0.703 \\
\hline AEe5 & 1.760 & 1.308 & 4.620 & 4.331 & 40.818 & 3.028 & 28.716 & 0.074 & 0.423 & 0.587 \\
\hline AEe6 & 1.941 & 1.852 & 5.197 & 4.531 & 46.482 & 4.598 & 30.694 & 0.099 & 0.460 & 0.514 \\
\hline \multicolumn{11}{|c|}{ Sequence AFe: $f_{\mathrm{sh}}=0.1 ; f_{\mathrm{K}}=0.95 ; f_{\mathrm{p}}=0.10 ; a=1.2 ; \rho_{\mathrm{c}}=2 \times 10^{9} \mathrm{~g} \mathrm{~cm}^{-3}$} \\
\hline AFe2 & 1.393 & 0.175 & 0.577 & 1.242 & 29.657 & 0.136 & 24.459 & 0.005 & 0.379 & 0.775 \\
\hline AFe6 & 1.481 & 0.526 & 2.887 & 2.990 & 32.231 & 0.787 & 25.469 & 0.024 & 0.438 & 0.638 \\
\hline AFe8 & 1.582 & 0.825 & 4.042 & 3.723 & 35.252 & 1.567 & 26.630 & 0.044 & 0.495 & 0.536 \\
\hline $\mathrm{AFe} 9$ & 1.664 & 1.066 & 4.620 & 4.017 & 37.684 & 2.222 & 27.519 & 0.059 & 0.542 & 0.478 \\
\hline $\mathrm{AFe} 10$ & 1.784 & 1.431 & 5.197 & 4.203 & 41.208 & 3.201 & 28.748 & 0.078 & 0.610 & 0.406 \\
\hline $\mathrm{AFe} 11$ & 1.939 & 1.941 & 5.775 & 4.226 & 45.746 & 4.501 & 30.247 & 0.098 & 0.689 & 0.348 \\
\hline $\mathrm{AFe} 12$ & 2.046 & 2.318 & 6.006 & 4.190 & 48.912 & 5.439 & 31.263 & 0.111 & 0.741 & 0.312 \\
\hline \multicolumn{11}{|c|}{ Sequence AGe: $f_{\mathrm{sh}}=-1.0 ; f_{\mathrm{K}}=0.6 ; f_{\mathrm{p}}=0.01 ; a=0.9 ; \rho_{\mathrm{c}}=2 \times 10^{9} \mathrm{~g} \mathrm{~cm}^{-3}$} \\
\hline AGe1 & 1.524 & 0.630 & 5.775 & 3.448 & 33.996 & 1.121 & 26.391 & 0.033 & 0.365 & 0.732 \\
\hline AGe3 & 1.585 & 0.811 & 6.237 & 3.838 & 35.821 & 1.606 & 27.069 & 0.045 & 0.396 & 0.659 \\
\hline AGe5 & 1.657 & 1.032 & 6.699 & 4.073 & 37.862 & 2.181 & 27.764 & 0.058 & 0.438 & 0.580 \\
\hline AGe6 & 1.730 & 1.269 & 6.930 & 4.127 & 39.786 & 2.754 & 28.362 & 0.069 & 0.486 & 0.514 \\
\hline AGe7 & 1.803 & 1.527 & 7.045 & 4.036 & 41.535 & 3.306 & 28.845 & 0.080 & 0.541 & 0.457 \\
\hline AGe8 & 1.890 & 1.881 & 7.161 & 3.771 & 43.371 & 3.934 & 29.262 & 0.091 & 0.616 & 0.391 \\
\hline \multicolumn{11}{|c|}{ Sequence Re: Rigid Rotation; $\rho_{\mathrm{c}}=2 \times 10^{9} \mathrm{~g} \mathrm{~cm}^{-3}$} \\
\hline Re1 & 1.376 & 0.000 & 0.000 & 0.000 & 29.248 & 0.000 & 24.356 & 0.000 & 0.296 & 1.000 \\
\hline Re4 & 1.392 & 0.180 & 1.309 & 1.309 & 29.715 & 0.118 & 24.540 & 0.004 & 0.306 & 0.957 \\
\hline Re6 & 1.415 & 0.294 & 2.004 & 2.004 & 30.399 & 0.294 & 24.802 & 0.010 & 0.326 & 0.884 \\
\hline $\operatorname{Re} 7$ & 1.428 & 0.344 & 2.269 & 2.269 & 30.765 & 0.391 & 24.938 & 0.013 & 0.340 & 0.848 \\
\hline $\operatorname{Re} 8$ & 1.440 & 0.388 & 2.472 & 2.472 & 31.095 & 0.480 & 25.059 & 0.015 & 0.357 & 0.797 \\
\hline $\operatorname{Re} 9$ & 1.449 & 0.422 & 2.613 & 2.613 & 31.355 & 0.551 & 25.151 & 0.018 & 0.377 & 0.754 \\
\hline Re10 & 1.455 & 0.443 & 2.694 & 2.694 & 31.519 & 0.597 & 25.208 & 0.019 & 0.400 & 0.710 \\
\hline $\operatorname{Re} 11$ & 1.458 & 0.451 & 2.724 & 2.724 & 31.579 & 0.615 & 25.228 & 0.019 & 0.426 & 0.667 \\
\hline
\end{tabular}



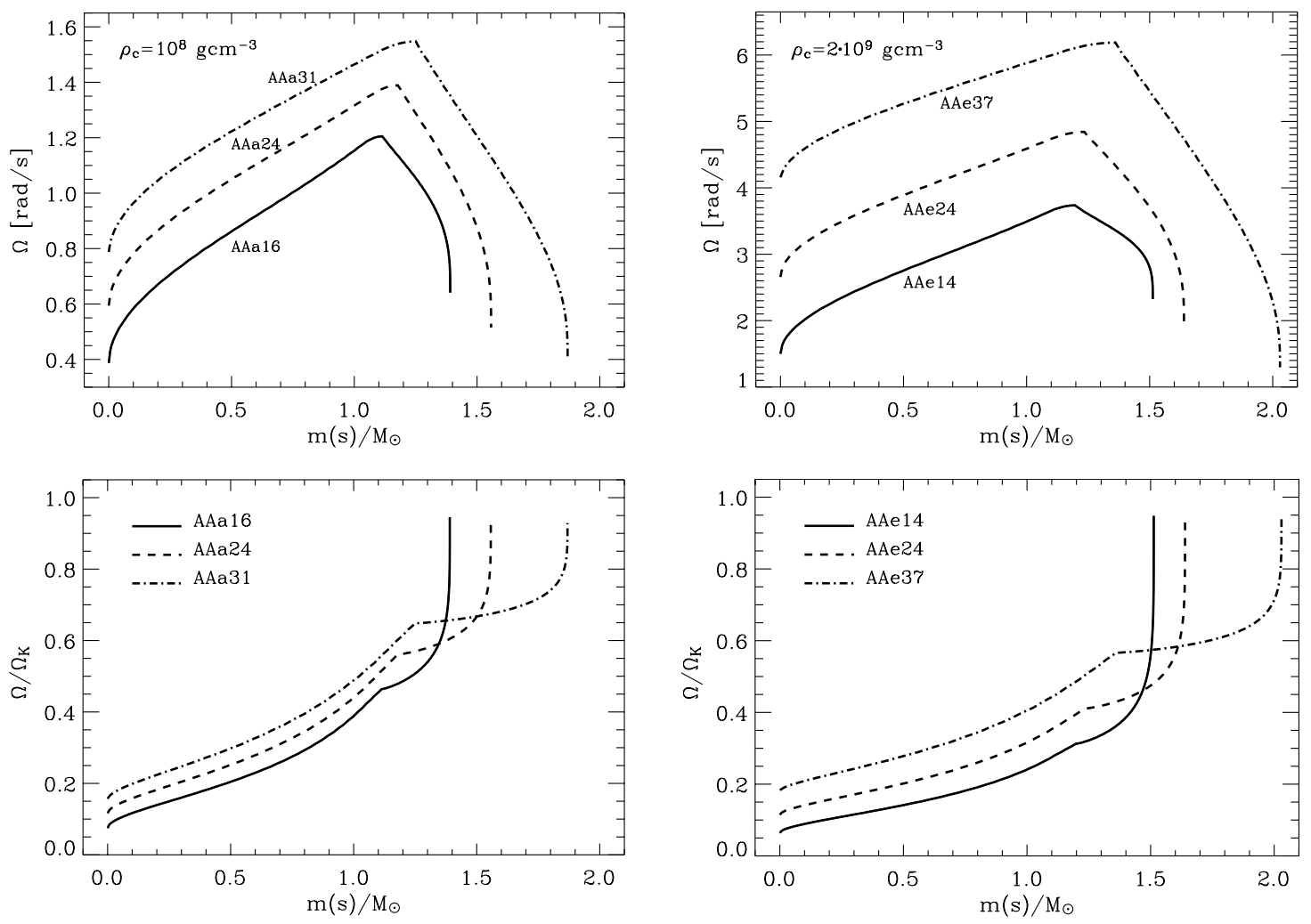

Fig. 1. Upper panels: angular velocity as a function of the mass coordinate in white dwarf models (AAa16, AAa24 and AAa31 in the left panel, and AAe10, AAe24 and AAe37 in the right panel). Lower panels: angular velocity normalized to the local Keplerian value as a function of the mass coordinate, in the corresponding models.

white dwarf models with different values of $\Omega_{\mathrm{c}}$ are constructed. Since the mass budget in binary systems considered for the Single Degenerate scenario for SNe Ia is limited, the maximum possible mass that a white dwarf can achieve by mass accretion cannot significantly exceed $2.0 M_{\odot}$ (Langer et al. 2000). Therefore, we do not construct models with $M \gtrsim 2.1 M_{\odot}$.

\section{Basic model properties}

Properties of selected models with the AWD rotation law are presented in Tables 1 and 2. As mentioned earlier, $\Omega$ profiles in the models with $f_{\mathrm{sh}}=1.0, f_{\mathrm{p}}=0.05$ or 0.1 , and $f_{\mathrm{K}}=0.95$ (Seqs. AA, AB, \& AC) resemble closest the $1 \mathrm{D}$ models in Paper I. The models with $\rho_{\mathrm{c}}=2 \times 10^{9} \mathrm{~g} \mathrm{~cm}^{-3}$ represent the pre-explosion stages, since carbon burning is expected to occur at roughly this density in accreting white dwarfs with $\dot{M} \gtrsim 10^{-7} M_{\odot} \mathrm{yr}^{-1}$ (e.g. Nomoto 1982).

Figure 1 shows angular velocity profiles of six different models, as indicated. Here, the mass coordinate $m(s)$ is defined such that $m(s)=2 \pi \int_{0}^{s} \int_{-\infty}^{\infty} \rho\left(s^{\prime}, z\right) \mathrm{d} z \mathrm{~d} s^{\prime}$. These profiles have similar shapes to those in $1 \mathrm{D}$ white models in Paper I. A detailed comparison to 1D models is performed in Sect. 5. Note that models with $\rho_{\mathrm{c}}=2 \times 10^{9} \mathrm{~g} \mathrm{~cm}^{-3}$ have higher values of $\Omega$ than those with $\rho_{\mathrm{c}}=1 \times 10^{8} \mathrm{~g} \mathrm{~cm}^{-3}$ at a given mass, due to their stronger compactness. This tendency is reflected in the values of the moment-of-inertia weighted mean of $\Omega$ in Table 1 .

Iso-density contours of the corresponding models with $\rho_{\mathrm{c}}=$ $2 \times 10^{9} \mathrm{~g} \mathrm{~cm}^{-3}$ are given in Fig. 2. As the value of $T / W$ increases, the white dwarf structure deviates from the spheroidal shape more and more, and the ratio of the polar radius to the equatorial radius decreases. However, the inner core with $\rho \gtrsim 0.05 \rho_{\mathrm{c}}$, which contains more than $75 \%$ of the total mass, remains fairly spheroidal even when $T / W=0.11$.

Even though our 2D models are designed to resemble the results from evolutionary calculations, the parameter range which describes them is finite due to two effects. Firstly, variations in the evolution itself, i.e. of the accretion rate in binary systems, or of the initial white dwarf mass, give rise to variations, say, in the pre-supernova modes (i.e., models with $\left.\rho_{\mathrm{c}}=2 \times 10^{9} \mathrm{~g} \mathrm{~cm}^{-3}\right)$; i.e., the value of $f_{\mathrm{p}}$ was shown to depend on both in Paper I. Secondly, some physical processes described in the evolutionary models are uncertain. For instance, the exact value of the critical Richardson number is still uncertain, and instead of $R_{\mathrm{i}, \mathrm{c}}=0.25$ that is used in the present study, $R_{\mathrm{i}, \mathrm{c}}=1.0$ is also often adopted in the literature (e.g. Hirschi et al. 2004), with which the shear rate in the inner core of white dwarfs would become weaker than assumed in sequence AA. Therefore, we constructed 2D models in which we varied all relevant parameters (Table 2).

Figure 3 shows $\Omega$-profiles in 5 different models (AAe20, $\mathrm{ABe} 23, \mathrm{ACe} 19, \mathrm{AFe} 8$ and AG3), which have the same central density $\left(\rho_{\mathrm{c}}=2 \times 10^{9} \mathrm{~g}, \mathrm{~cm}^{-3}\right)$ and similar masses $\left(\sim 1.58 M_{\odot}\right)$, but different sets of $f_{\mathrm{p}}, f_{\mathrm{sh}}, f_{\mathrm{K}}$ and $a$ (see Tables 1 and 2 ). A comparison of model AAe20 with model ABe23 shows the influence of $f_{\mathrm{p}}$. For smaller $f_{\mathrm{p}}$, the $\Omega$-peak is located 

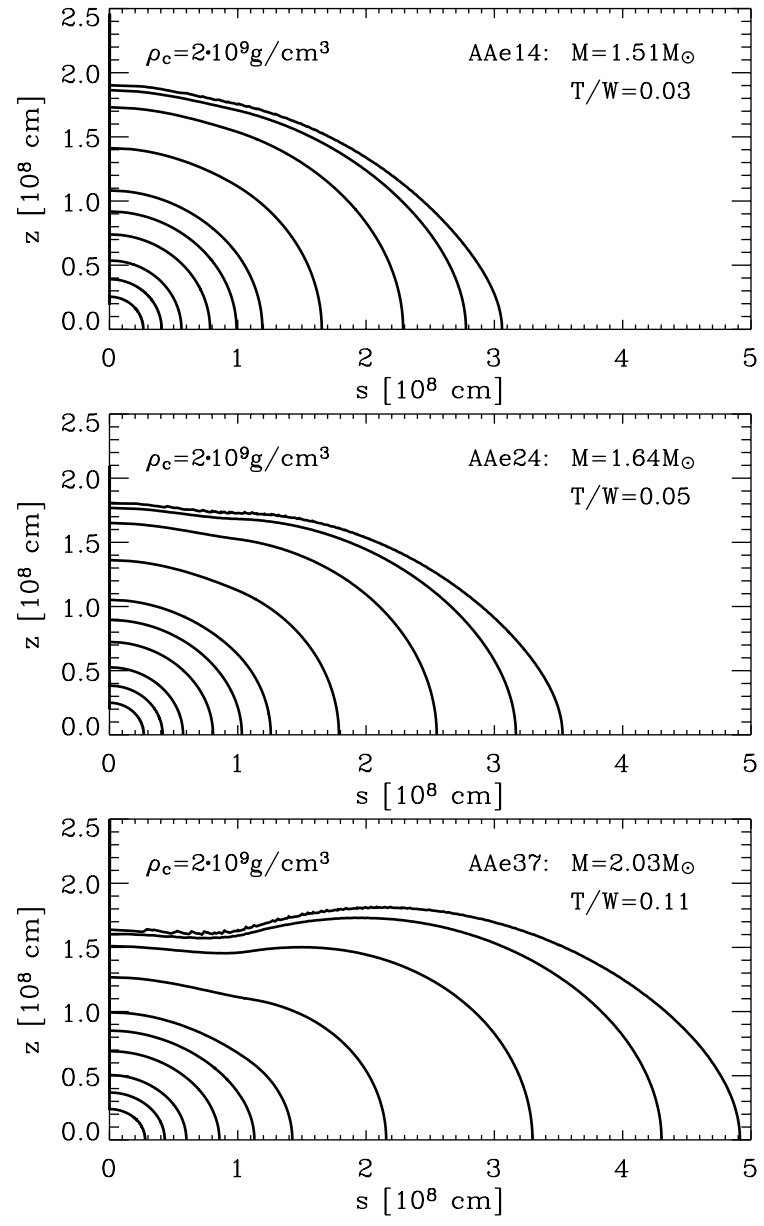

Fig. 2. Iso-density contours of white dwarf models AAe14, AAe24 and AAe37. These models have the same central density of $\rho_{\mathrm{c}}=2 \times$ $10^{9} \mathrm{~g} \mathrm{~cm}^{-3}$, but different masses and angular momenta. The contour lines denote levels of $0.8 \rho_{\mathrm{c}}, 0.6 \rho_{\mathrm{c}}, 0.4 \rho_{\mathrm{c}}, 0.2 \rho_{\mathrm{c}}, 0.1 \rho_{\mathrm{c}}, 0.05 \rho_{\mathrm{c}}, 10^{-2} \rho_{\mathrm{c}}$, $10^{-3} \rho_{\mathrm{c}}, 10^{-4} \rho_{\mathrm{c}}$ and $10^{-5} \rho_{\mathrm{c}}$, from the central region to the outer layers.

further outward. As a result, the inner core rotates more slowly and the outer region more rapidly, for fixed mass and central density. The influence of $a$ can be understood by comparing model AAe20 with model ACe19: the surface region rotates more rapidly and the inner core more slowly in model ACe19. This is because, with a smaller $a$, the role of the centrifugal force becomes stronger in the region of $s>s_{\mathrm{p}}$ (see Eq. (8)). Model AFe8 has a weaker shear rate in the inner core than model AAe20. As a consequence, this model needs a higher value of $\Omega_{\mathrm{c}}$ than model AAe20, to retain the same central density at a given mass. In model AGe3, where the $\Omega$-gradient is negative throughout the white dwarf, the inner core rotates much faster than the other models. In Fig. 4, models with $j$-constant and $v$-constant rotation laws are compared with models AAe20 and AGe3. Note that differential rotation in those models is much stronger than in model AGe30, which means that the shear rates for such exotic rotation laws are well above the critical value for the onset of the dynamical shear instability, $\sigma_{\text {DSI,crit }}$.

The specific angular momentum profiles in Fig. 3 show an interesting feature. If a white dwarf model has lower values of $j$
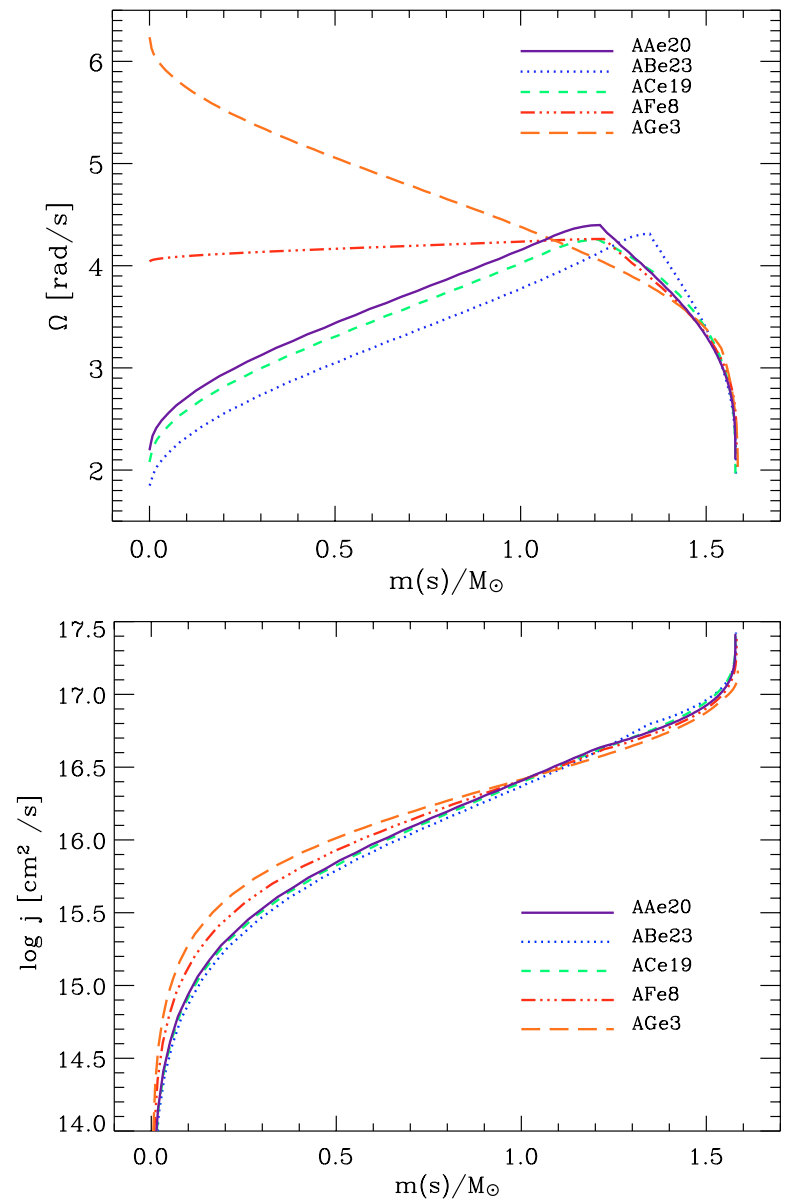

Fig. 3. Upper panel: angular velocity as a function of the mass coordinate in white dwarf models AAe20 (solid line), ABe20 (dotted line), ACe19 (short-dashed), AFe8 (dashed-three-dotted line) and AGe3 (long-dashed line). Lower panel: specific angular momentum in the corresponding models of the upper panel.

in the inner core than the other models do, it has higher values of $j$ in the outer layers. As remarkable consequence, all the white dwarf models displayed in Fig. 3 have a similar amount of total angular momentum (see Tables 1 and 2), despite their different angular momentum distributions. Figure 5, where the total angular momentum $J$ of models in different model sequences (AAe, $\mathrm{ABe}, \mathrm{ACe}, \mathrm{AFe}, \mathrm{AGe}$ and $\mathrm{Re}$ ) is plotted in the $M-J$ plane, indeed shows that the total angular momentum, for a given central density, is a function of the white dwarf mass only, regardless of various possible rotation profiles. Although the scatter in the value of $J$ becomes larger as the white dwarf mass increases, it remains within $10 \%$ of the mean value even when $M \gtrsim 1.9 M_{\odot}$. Even the rigidly rotating white dwarf models $(M \lesssim 1.47)$ follow the general trend very well. We conclude that the $J-M$-law predicted by our models is not sensitive to evolutionary details or uncertain physical processes.

For comparison, we also give results with $j$-constant and $v$-constant rotation laws in the same $M-J$ plane. Note that models with these exotic rotation laws follow very different relations. However, as mentioned above, these models are shear unstable, and their steep angular velocity gradients should decay on a short time scale (see Paper I). Therefore, we can 


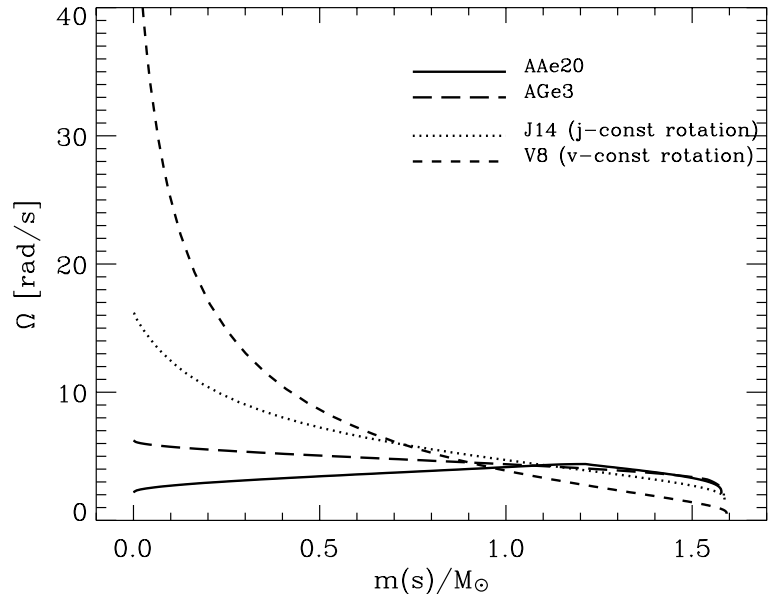

Fig. 4. Angular velocity as a function of the mass-coordinate in models AAe20 (solid line), AGe3 (long-dashed line), J14 (dotted line) and V8 (short-dashed line).

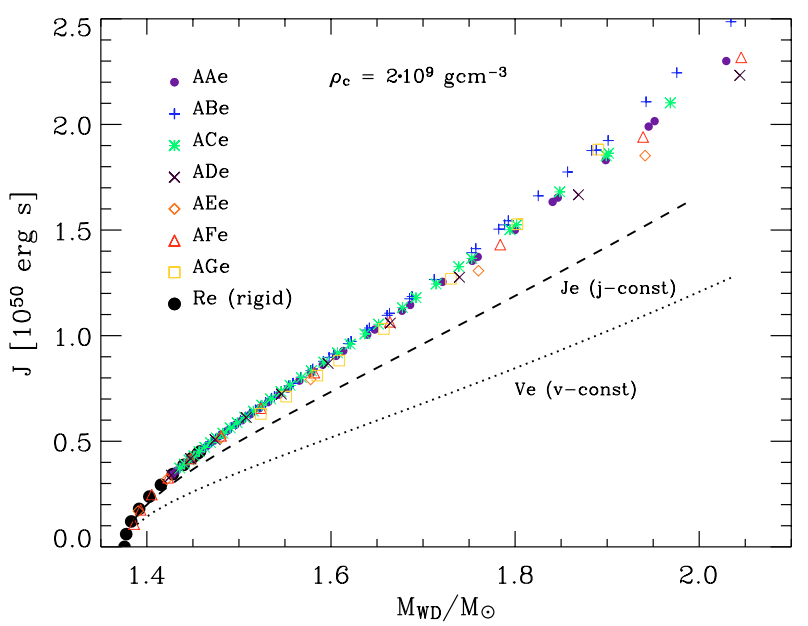

Fig. 5. Total angular momentum of white dwarf models as a function of the total mass of the white dwarf, in sequences $\mathrm{AAe}, \mathrm{ABe}, \mathrm{ACe}$, $\mathrm{AFe}$ and $\mathrm{AGe}$.

conclude that the $M-J$ relation is not significantly affected by different $\Omega$-profiles in general. We find that this is also the case for the dependence of other physical quantities on the total mass, such as for $T / W$ and and for the binding energy, as discussed in the following section.

\section{Functional relations of physical quantities}

Here we derive analytical expressions for various physical quantities as functions of the white dwarf mass $(M)$ and the central density $\left(\rho_{\mathrm{c}}\right)$, from models with the AWD-rotation and rigid rotation laws.

\section{1. $M-J$ relation}

Figure 6 shows the total angular momentum of white dwarf models with the AWD or rigid rotation law for 7 different central densities, in the $M-J$ plane. White dwarfs with lower central densities have systematically larger angular momenta at a given mass, as expected from the role of the centrifugal
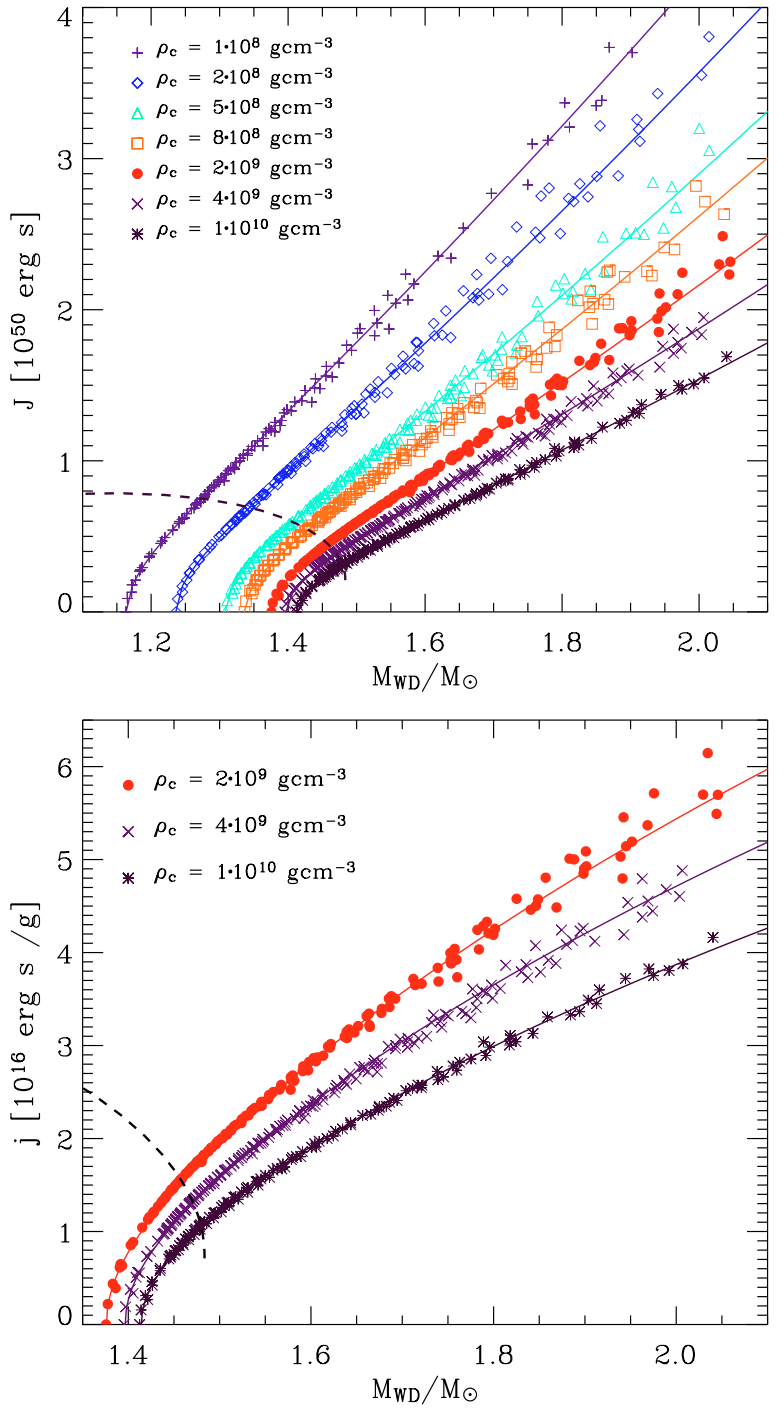

Fig. 6. Upper panel: total angular momentum of white dwarf models with the AWD rotation and rigid rotation laws, as a function of the white dwarf mass. Models at different central densities are plotted with different symbols as indicated in the figure. The solid lines denote the fitting function given by Eq. (19). The dashed line gives the $M-J$ relation of rigidly rotating white dwarfs at critical rotation. Lower panel: specific angular momentum of white dwarf models with the AWD rotation and rigid rotation laws, as a function of the white dwarf mass. Models with $\rho_{\mathrm{c}}=2 \times 10^{9} \mathrm{~g} \mathrm{~cm}^{-3}, 4 \times 10^{9} \mathrm{~g} \mathrm{~cm}^{-3}$ and $10^{10} \mathrm{~g} \mathrm{~cm}^{-3}$ are plotted with different symbols as indicated in the figure. The solid lines represent $J\left(M ; \rho_{\mathrm{c}}\right) / M$, where $J\left(M ; \rho_{\mathrm{c}}\right)$ is given by Eq. (19). The dashed line gives the $M-j$ relation of rigidly rotating white dwarfs at critical rotation.

force. We find that the relation between the total angular momentum and the total mass of the white dwarf can be given in an analytical form, in the considered range of the central density $\left(10^{8} \lesssim \rho_{\mathrm{c}}\left(\mathrm{g} \mathrm{cm}^{-3}\right) \lesssim 10^{10}\right)$ and the white dwarf mass $\left(M \lesssim 2.0 M_{\odot}\right)$, as

$$
\begin{aligned}
J\left(M ; \rho_{\mathrm{c}}\right)= & C_{1}\left(\rho_{\mathrm{c}}\right)\left\{1-\exp \left(-0.2\left[M-M_{\mathrm{NR}}\left(\rho_{\mathrm{c}}\right)\right]^{0.48}\right)\right\} \\
& +C_{2}\left(\rho_{\mathrm{c}}\right)\left[M-M_{\mathrm{NR}}\left(\rho_{\mathrm{c}}\right)\right]^{1.3}\left[10^{50} \mathrm{erg} \mathrm{s}\right],
\end{aligned}
$$


where

$C_{1}\left(\rho_{\mathrm{c}}\right)=20.800370-1.5856256 \log _{10} \rho_{\mathrm{c}}$,

and

$$
C_{2}\left(\rho_{\mathrm{c}}\right)=11.397626-0.97306637 \log _{10} \rho_{\mathrm{c}} .
$$

Here, $M_{\mathrm{NR}}\left(\rho_{\mathrm{c}}\right)$ denotes the mass of the non-rotating white dwarf at a given central density, and both $M$ and $M_{\mathrm{NR}}$ are given in units of solar masses. From non-rotating white dwarf models, we derive $M_{\mathrm{NR}}$ as a function of $\rho_{\mathrm{c}}$ as

$$
\begin{aligned}
M_{\mathrm{NR}}= & 1.436 \\
& \times\left[1-\exp \left\{-0.01316\left(\log _{10} \rho_{\mathrm{c}}\right)^{2.706}+0.2493 \log _{10} \rho_{\mathrm{c}}\right\}\right] \\
& \left(\text { for } \rho_{\mathrm{c}}>10^{7} \mathrm{~g} \mathrm{~cm}^{-3}\right) .
\end{aligned}
$$

We are particularly interested in the cases of $\rho_{\mathrm{c}}=2 \times$ $10^{9} \mathrm{~g} \mathrm{~cm}^{-3}, \rho_{\mathrm{c}}=4 \times 10^{9} \mathrm{~g} \mathrm{~cm}^{-3}$ and $10^{10} \mathrm{~g} \mathrm{~cm}^{-3}$, as they represent critical densities for the thermonuclear explosion of $\mathrm{CO}$ white dwarfs, and the electron-capture induced collapse of $\mathrm{ONeMg}$ and $\mathrm{CO}$ white dwarfs, respectively (see Nomoto \& Kondo 1991). Hereafter, we refer to the total angular momentum at these densities as $J_{\mathrm{SNI}}, J_{\mathrm{EC}, \mathrm{ONM}}$, and $J_{\mathrm{EC}, \mathrm{CO}}$, respectively:

$J_{\mathrm{SNIa}}=J\left(M ; \rho_{\mathrm{c}}=2 \times 10^{9} \mathrm{~g} \mathrm{~cm}^{-3}\right)$,

$J_{\mathrm{EC}, \mathrm{ONM}}=J\left(M ; \rho_{\mathrm{c}}=4 \times 10^{9} \mathrm{~g} \mathrm{~cm}^{-3}\right)$,

and

$J_{\mathrm{EC}, \mathrm{CO}}=J\left(M ; \rho_{\mathrm{c}}=10^{10} \mathrm{~g} \mathrm{~cm}^{-3}\right)$.

If a $\mathrm{CO}$ white dwarf has a larger amount of angular momentum than $J_{\text {SNIa }}$, a SN Ia explosion cannot be induced. Similarly, an $\mathrm{ONeMg}$ white dwarf with $J>J_{\mathrm{EC} \text {,ONM }}$ will not experience electron-capture induced collapse. On the other hand, a SN Ia explosion is expected when $J_{\mathrm{EC}, \mathrm{CO}} \lesssim J \lesssim J_{\mathrm{SNIa}}$. If carbon ignites at the center when $J<J_{\mathrm{EC}, \mathrm{CO}}$, the outcome will be electron capture induced collapse. These criteria for SN Ia explosion and EC induced collapse are summarized in Fig. 7.

Alternatively, the critical $\mathrm{CO}$ white dwarf mass for a SN Ia explosion $\left(M_{\mathrm{SNIa}}\right)$ and $\mathrm{ONeMg}$ or $\mathrm{CO}$ white dwarf mass for EC induced collapse $\left(M_{\mathrm{EC}, \mathrm{ONM}}\right.$ or $\left.M_{\mathrm{EC}, \mathrm{CO}}\right)$ can be given as a function of $J$, for $M \lesssim 2.0 M_{\odot}$, as

$$
\begin{aligned}
& M_{\mathrm{SNIa}}=1.376-0.024 J^{0.83}+1-\exp \left(-0.330 J^{1.5}\right), \\
& M_{\mathrm{EC}, \mathrm{ONM}}=1.395-0.040 J^{0.83}+1-\exp \left(-0.416 J^{1.5}\right),
\end{aligned}
$$

and

$$
M_{\mathrm{EC}, \mathrm{CO}}=1.412-0.042 J^{0.83}+1-\exp \left(-0.519 J^{1.5}\right) .
$$

I.e., exploding/collapsing white dwarfs are expected to have roughly these critical masses for a given total amount of angular momentum.

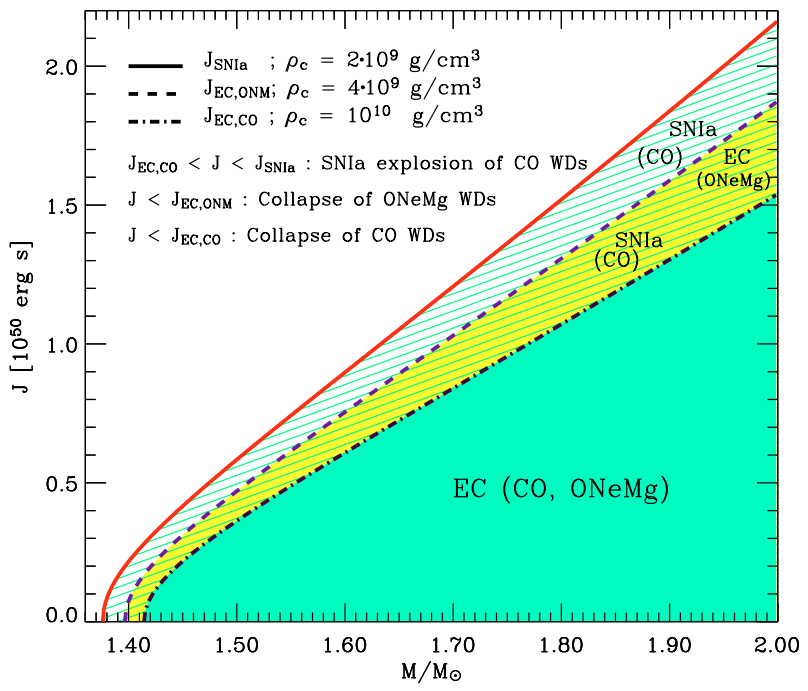

Fig. 7. The critical angular momentum for thermonuclear explosion of $\mathrm{CO}$ white dwarfs ( $J_{\mathrm{SNIa}}$; solid line), and electron-capture induced collapse of $\mathrm{CO}$ white dwarfs ( $J_{\mathrm{EC}, \mathrm{CO}}$; dashed-dotted line) and $\mathrm{ONeMg}$ white dwarfs ( $J_{\mathrm{EC}, \mathrm{ONM}}$; dashed line), as a function of the white dwarf mass. SNIa explosion is expected when $J_{\mathrm{EC}, \mathrm{CO}} \lesssim J \lesssim J_{\mathrm{SNIa}}$ (hatched region). Electron-capture induced collapse is supposed to occur when $J \lesssim J_{\mathrm{EC}, \mathrm{CO}}$ for CO white dwarfs, and $J \lesssim J_{\mathrm{EC}, \mathrm{ONeMg}}$ for ONeMg white dwarfs, respectively.

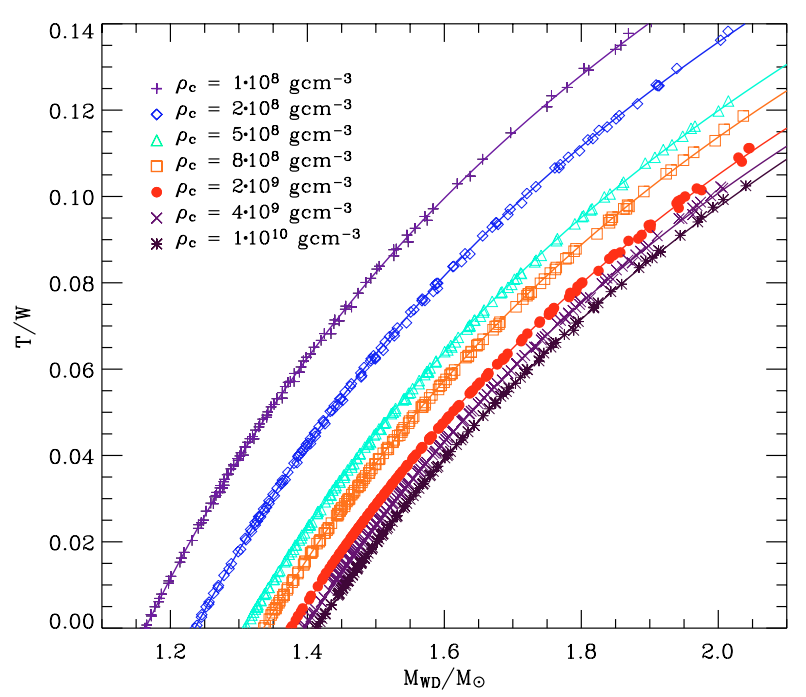

Fig. 8. Ratio of the rotational energy to the gravitational energy $(T / W)$ in white dwarf models with the AWD rotation and rigid rotation laws, as a function of the white dwarf mass. Models at different central densities are plotted with different symbols as indicated in the figure. The solid lines denote the fitting function given by Eq. (29).

\section{2. $M-T / W$ relation}

The ratio of the rotational energy to the gravitational energy $(T / W)$ is plotted as a function of the white dwarf mass in Fig. 8, for models with the AWD rotation and rigid rotation laws. For a fixed central density $\rho_{\mathrm{c}}$, the values of $T / W$ follow very well a one-dimensional relation; the scatter is much smaller than in the corresponding diagram showing the total 
angular momentum (Fig. 6). This relation can be given as a function of $M$ and $\rho_{\mathrm{c}}$, as

$$
\begin{aligned}
T / W\left(M ; \rho_{\mathrm{c}}\right)= & 0.1\left\{1-\exp \left(-C_{3}\left(\rho_{\mathrm{c}}\right)\left[M-M_{\mathrm{NR}}\left(\rho_{\mathrm{c}}\right)\right]\right)\right\} \\
& +C_{4}\left(\rho_{\mathrm{c}}\right)\left[M-M_{\mathrm{NR}}\left(\rho_{\mathrm{c}}\right)\right]
\end{aligned}
$$

where

$$
\begin{aligned}
C_{3}\left(\rho_{\mathrm{c}}\right)= & 407.71741-168.99280 \log _{10} \rho_{\mathrm{c}} \\
& +26.449872\left(\log _{10} \rho_{\mathrm{c}}\right)^{2}-1.8421546\left(\log _{10} \rho_{\mathrm{c}}\right)^{3} \\
& +0.048121239\left(\log _{10} \rho_{\mathrm{c}}\right)^{4}
\end{aligned}
$$

and

$$
\begin{aligned}
C_{4}\left(\rho_{\mathrm{c}}\right)= & -3.2305060+1.5464029 \log _{10} \rho_{\mathrm{c}} \\
& -0.26049998\left(\log _{10} \rho_{\mathrm{c}}\right)^{2}+0.018811083\left(\log _{10} \rho_{\mathrm{c}}\right)^{3} \\
& -0.00049405568\left(\log _{10} \rho_{\mathrm{c}}\right)^{4} .
\end{aligned}
$$

Note that, in the considered mass range $\left(M \lesssim 2.0 M_{\odot}\right)$, only the models with $\rho_{\mathrm{c}}=1 \times 10^{8} \mathrm{~g} \mathrm{~cm}^{-3}$ and $2 \times 10^{8} \mathrm{~g} \mathrm{~cm}^{-3}$ reach the canonical value of 0.14 for the onset of the bar-mode instability (e.g. Durisen \& Imamura 1977).

\subsection{Binding energy}

The binding energy $(B E:=W-U-T)$ is essential for the energy budget of exploding or collapsing white dwarfs. Figure 9 shows binding energy and specific binding energy of our white dwarf models with $\rho_{\mathrm{c}}=2 \times 10^{9} \mathrm{~g} \mathrm{~cm}^{-3}$ and $\rho_{\mathrm{c}}=10^{10} \mathrm{~g} \mathrm{~cm}^{-3}$, which may represent the pre-explosion and pre-collapse stages of $\mathrm{CO}$ white dwarfs, respectively, and with $4 \times 10^{9} \mathrm{~g} \mathrm{~cm}^{-3}$, which refers to the pre-collapse stage of $\mathrm{ONeMg}$ white dwarfs. Both binding energy and specific binding energy are monotonically increasing functions of the white dwarf mass.

We constructed a fitting formula for the binding energy as a function of the white dwarf mass, which is applicable in the considered range of central densities $\left(1 \times 10^{8} \lesssim \rho_{\mathrm{c}}\left[\mathrm{g} \mathrm{cm}^{-3}\right] \lesssim\right.$ $\left.10^{10}\right)$ :

$$
\begin{aligned}
B E\left(M ; \rho_{\mathrm{c}}\right)= & B E_{\mathrm{NR}}\left(\rho_{\mathrm{c}}\right) \\
& +C_{5}\left(\rho_{\mathrm{c}}\right)\left[M-M_{\mathrm{NR}}\left(\rho_{\mathrm{c}}\right)\right]^{1.03} \quad\left[10^{50} \mathrm{erg}\right],
\end{aligned}
$$

where

$$
\begin{aligned}
C_{5}\left(\rho_{\mathrm{c}}\right)= & -370.73052+132.97204 \log _{10} \rho_{\mathrm{c}} \\
& -16.117031\left(\log _{10} \rho_{\mathrm{c}}\right)^{2}+0.66986678\left(\log _{10} \rho_{\mathrm{c}}\right)^{3} .
\end{aligned}
$$

The binding energy of non-rotating white dwarfs $B E_{\mathrm{NR}}$ is given by

$$
\begin{aligned}
B E_{\mathrm{NR}}\left(\rho_{\mathrm{c}}\right)= & -32.759747+6.7179802 \log _{10} \rho_{\mathrm{c}} \\
& -0.28717609\left(\log _{10} \rho_{\mathrm{c}}\right)^{2} \quad\left[10^{50} \mathrm{erg}\right] .
\end{aligned}
$$
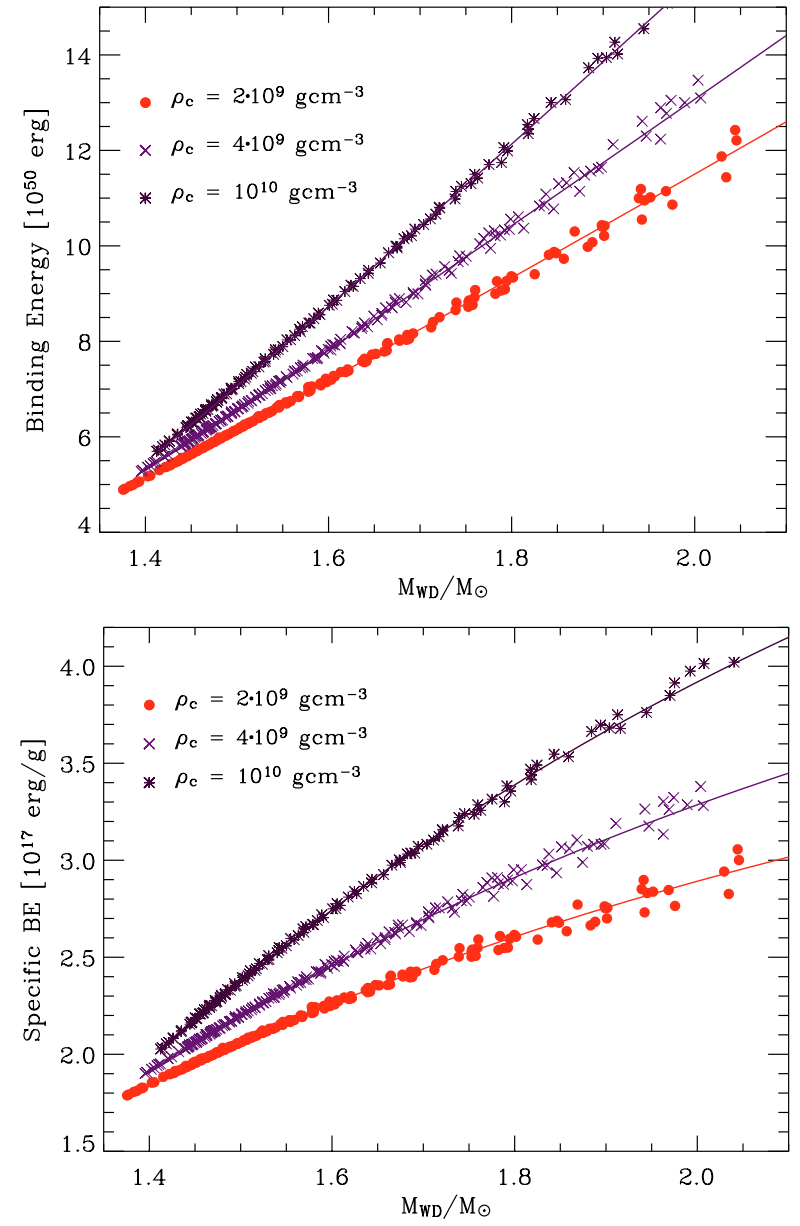

Fig. 9. Upper panel: binding energy of white dwarf models with the AWD rotation and rigid rotation laws for $\rho_{\mathrm{c}}=2 \times 10^{9} \mathrm{~g} \mathrm{~cm}^{-3} 4 \times$ $10^{9} \mathrm{~g} \mathrm{~cm}^{-3}$, and $10^{10} \mathrm{~g} \mathrm{~cm}^{-3}$, as function of the white dwarf mass. The solid lines denote the fitting function given by Eq. (32). Lower panel: specific binding energy of white dwarf models with the AWD rotation and rigid rotation laws for $\rho_{\mathrm{c}}=2 \times 10^{9} \mathrm{~g} \mathrm{~cm}^{-3}, 4 \times 10^{9} \mathrm{~g} \mathrm{~cm}^{-3}$, and $10^{10} \mathrm{~g} \mathrm{~cm}^{-3}$. The solid lines are from the fitting function given by Eq. (32), divided by $M$.

\section{Comparison with 1D models}

In Fig. 10, we compare our 2D results with two time-dependent 1D model sequences of Paper $\mathrm{I}$ in the $M-T / W$ and $M-$ $J$ planes. These sequences simulate white dwarfs which accrete with constant accretion rates of $5 \times 10^{-7} M_{\odot} \mathrm{yr}^{-1}$, for an initial white dwarf mass of $0.8 M_{\odot}$ (sequence C2) and $1.0 M_{\odot}$ (sequence $\mathrm{C} 10$ ). Figure 10 shows that the models of sequence $\mathrm{C} 10$ have similar $T / W$ values at a given mass and a central density as the $2 \mathrm{D}$ models as long as $M \lesssim 1.6$. However, they deviate significantly for higher masses. The models of sequence $\mathrm{C} 2$, which have a higher angular momentum at a given mass compared to those of sequence $\mathrm{C} 10$, already deviate from the $2 \mathrm{D}$ models when $M \gtrsim 1.4$. We conclude that the $1 \mathrm{D}$ models cannot be regarded as accurate when $T / W \gtrsim 0.06$, which occurs after accreting about $0.4 M_{\odot}$ in both cases.

The inconsistency between $1 \mathrm{D}$ and 2D models at $T / W \gtrsim$ 0.06 is not surprising because in $1 \mathrm{D}$ models, the effect of the 

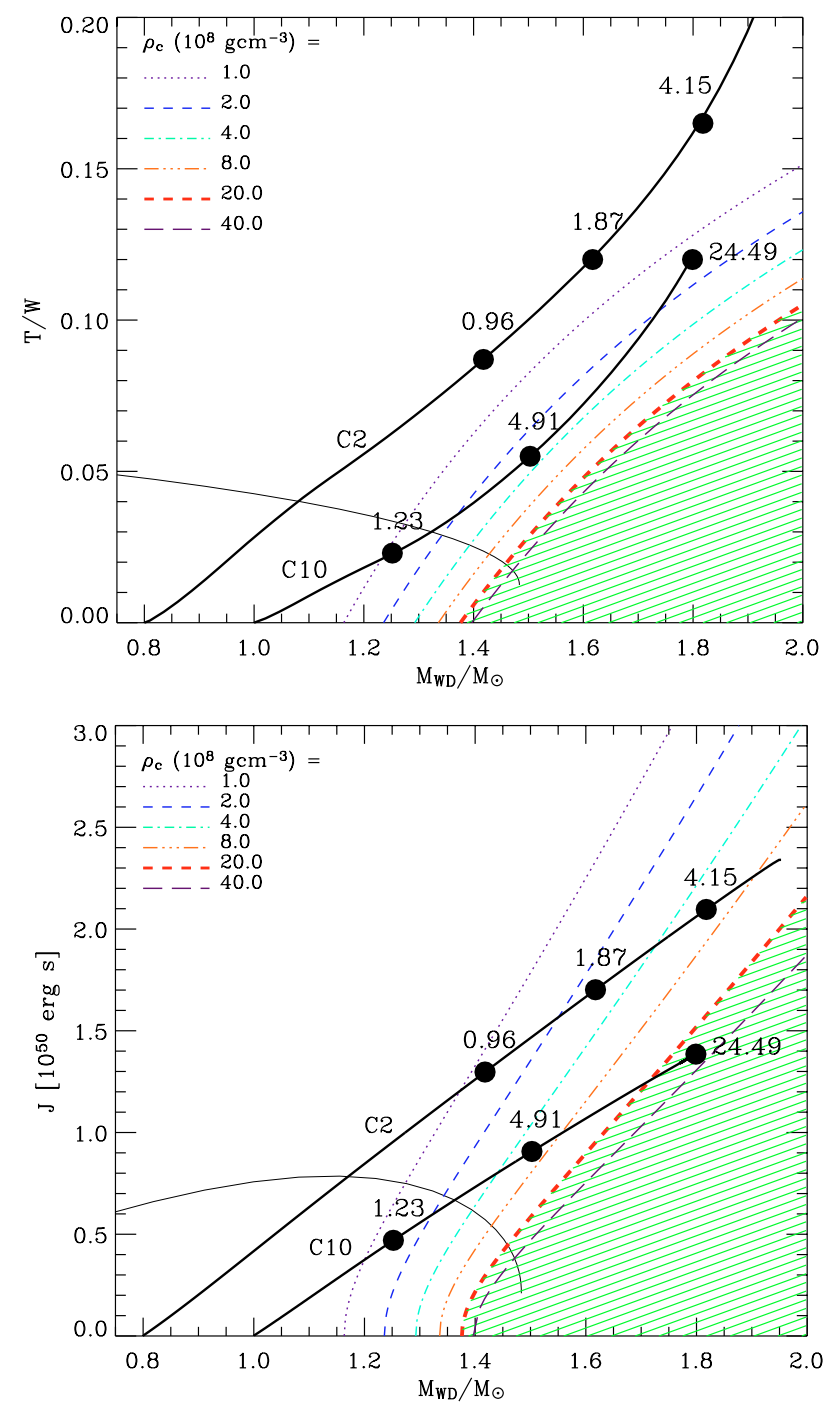

Fig. 10. Upper panel: ratio of rotational to gravitational energy $(T / W)$ as a function of the white dwarf mass. The thin solid line corresponds to the $M-T / W$ relation of rigidly rotating $2 \mathrm{D}$ white dwarf models at critical rotation. Thick solid lines are evolutionary tracks of the 1D sequences C2 and C10 in Paper I. Labels at filled circles on those tracks denote central densities in the unit of $10^{8} \mathrm{~g} \mathrm{~cm}^{-3}$. The $M-T / W$ relation given by Eq. (29) is also plotted for different densities: $\rho_{\mathrm{c}} /\left[10^{8} \mathrm{~g} \mathrm{~cm}^{-3}\right]=1.0$ (dotted line), 2.0 (dashed line), 4.0 (dashed-dotted line), 8.0 (dashed-three-dotted line), 20.0 (thick dashed line) and 40.0 (long-dashed line). Lower panel: same as in the upper panel, but in the $M-J$ plane.

centrifugal force on the white dwarf structure cannot be accurately described when a significant fraction of the white dwarf rotates at above $\sim 60 \%$ of the Keplerian value (cf. Paper I). In particular, the white dwarf radius $R$ is underestimated in this situation. In the $1 \mathrm{D}$ models, a certain amount of angular momentum is assumed to be accreted onto the white dwarf, and the consequent angular velocity is determined as $\Omega \sim J /\left(M R^{2}\right)$. From $T \sim M \Omega^{2} R^{2} \sim J^{2} /\left(M R^{2}\right)$ and $W \sim M^{2} / R$, we have $T / W \sim J^{2} /\left(M^{3} R\right)$. Therefore, at given $J$ and $M$, an underestimate of $R$ leads to an overestimate of $T / W$.

It is remarkable that $1 \mathrm{D}$ and $2 \mathrm{D}$ models show an extraordinary agreement in the $M-J$ plane throughout the considered mass range (Fig. 10, lower panel). However, the implication is not that 1D models can describe the white dwarf structure accurately. It should be rather considered as a coincidence for the following reason. The underestimate of $R$ in 1D models leads to an overestimate of $\rho_{\mathrm{c}}$, since $\rho_{\mathrm{c}} \sim M / R^{3}$. On the other hand, the central density should be reduced due to the overestimate of the centrifugal force, as the ratio of the centrifugal force to the gravitational force is given by $F_{\mathrm{c}} / F_{\mathrm{g}} \sim \Omega^{2} R /\left(G M / R^{2}\right) \sim$ $J^{2} /\left(M^{3} R\right)$. These two opposite effects might just compensate for each other.

In any case, the consistency between 1D and 2D models in the $M-J$ plane leads us to retain one of the most important conclusions of Paper I: If white dwarfs accrete mass and angular momentum efficiently, they might hardly reach central carbon ignition during the accretion phase.

\section{Implications for SNe la progenitor evolution}

Our 2D models of rotating white dwarfs show that various physical quantities can be given as a function of the white dwarf mass, as long as the shear rate inside the white dwarf is not stronger than the critical value for the onset of the DSI. In particular, the $M-J$ relations of white dwarfs at $\rho_{\mathrm{c}}=2 \times$ $10^{9} \mathrm{~g} \mathrm{~cm}^{-3}\left(J_{\text {SNIa }}\right)$ and $\rho_{\mathrm{c}}=10^{10} \mathrm{~g} \mathrm{~cm}^{-3}\left(J_{\mathrm{EC}, \mathrm{CO}}\right)$ provide a quantitative criterion for the supernova explosion of rotating white dwarfs, as summarized in Fig. 7. Here, we discuss possible evolutionary paths of accreting white dwarfs towards a supernova explosion.

\subsection{The angular momentum problem}

If a non-rotating white dwarf with the initial mass of $M_{\text {init }}$ accretes mass with a specific angular momentum of the Keplerian value (i.e., $j_{\mathrm{K}}=\sqrt{G M R}$ ), the total amount of accreted angular momentum when the white dwarf reaches mass $M_{\mathrm{f}}$ is

$$
\begin{aligned}
J & =\int j \mathrm{~d} M \approx \frac{2}{3} \sqrt{G R}\left[M_{\mathrm{f}}^{3 / 2}-M_{\text {init }}^{3 / 2}\right] \\
& \simeq 4 \times 10^{50}\left(\frac{R}{0.01 R_{\odot}}\right)^{1 / 2}\left[\left(\frac{M_{\mathrm{f}}}{M_{\odot}}\right)^{3 / 2}-\left(\frac{M_{\text {init }}}{M_{\odot}}\right)^{3 / 2}\right](\text { erg s }) .
\end{aligned}
$$

In binary systems considered for the so-called Single Degenerate scenario for SNe Ia, the mass budget is limited, and the maximum possible mass which a $\mathrm{CO}$ white dwarf can reach by mass accretion is about $2.0 M_{\odot}$ (Langer et al. 2000). Therefore, white dwarfs that are potentially able to perform a SN Ia explosion will have masses in the range of $1.4 \lesssim$ $M / M_{\odot} \lesssim 2.0$. Given that initial masses of $0.8 \ldots 1.0 M_{\odot}$ are usually considered in this context (e.g. Langer et al. 2000), the value of $J$ according to the above equation will reach $2.6 \ldots 8.1 \times$ $10^{50}$ erg s when the white dwarf mass reaches $1.4 \ldots 2.0 M_{\odot}$. This is certainly much larger than $J_{\text {SNIa }}$ (see Fig. 7), and central carbon ignition cannot occur in white dwarfs with such large angular momenta.

For accreting white dwarfs to end in a SN Ia, therefore, either smaller amounts of angular momentum need to be accreted, and/or a large fraction of the accreted angular 
momentum should be lost again. This means that the detailed evolutionary paths of SNe Ia progenitors will depend on the efficiency of the angular momentum gain and on the time scale of the angular momentum loss.

\subsection{Efficiency of the angular momentum gain}

In Paper I and also in Uenishi et al. (2003) and Saio \& Nomoto (2004), the outward angular momentum transport from the critically rotating star to the accretion disk is considered to alleviate the angular momentum problem, based on the work by Paczyński (1991) and Popham \& Narayan (1991). For instance, in Paper I, if the equatorial surface of a white dwarf model rotated at the Keplerian value, angular momentum was assumed to be transported outward from the white dwarf to the accretion disk, while otherwise angular momentum of a certain fraction of the Keplerian value was allowed to be accreted. However, the results of Paper I showed that even with this limitation, the efficiency of the angular momentum gain (hereafter, $\dot{J}_{\text {acc-efficiency) is usually too high to produce } \mathrm{SNe} \text { Ia during }}$ the accretion phase: only when the amount of the accreted angular momentum was limited to $30 \%$ of the Keplerian value could white dwarfs with a high initial mass $\left(M_{\text {init }}=1.0 M_{\odot}\right)$ reach carbon ignition at the center, which occurred at about $M \simeq 1.8 M_{\odot}$ (sequences $\mathrm{C} 10, \mathrm{C} 11$ and $\mathrm{C} 12$ in Paper I; see also Fig. 10).

It should be noted that the angular momentum accretion efficiency might be below 100\% on two accounts. Firstly, the models of Paper I neglected thermonuclear shell burning. Eddington-Sweet circulations will be induced in the presence of hydrogen and helium shell burning, which will accelerate the outward transport of angular momentum in the white dwarf envelope where the $\Omega$ gradient is negative. Accordingly, the surface of the white dwarf will rarely deviate from rotating critically, and the angular momentum gain from the accretion disk will be thus more severely restricted.

Secondly, as pointed out by Saio \& Nomoto (2004), the models of Paper I applied outward angular momentum transport to the accretion disk only for limiting the angular momentum gain when the white dwarf surface reaches critical rotation, but did not allow for the possibility of removing angular momentum from the white dwarf into the accretion disk. Simulations in Saio \& Nomoto (2004), who did consider the latter possibility, show that the total angular momentum in the accreting white dwarf cannot only increase, but also decrease, depending on the change in the surface conditions. Systematic studies of the angular momentum accretion efficiency are therefore highly desirable to improve our understanding of the evolution of SN Ia progenitors.

\subsection{Gravitational wave radiation}

As discussed in Paper I, rotating white dwarfs can be susceptible to the bar-mode or to the $r$-mode instability, which will lead to loss of angular momentum via gravitational wave radiation. Comparison of Figs. 6 and 8 indicates that $J \gtrsim 4 \times 10^{50} \mathrm{erg} \mathrm{s}$ is necessary for the white dwarf to reach $T / W=0.14$, which is the canonical value for the onset of the bar-mode instability. Although Paper I described 1D models with higher values of $T / W$, this is due to the overestimate of $T / W$ in the 1D approximation of the stellar structure, as discussed in Sect. 5. In terms of angular momentum, the 1D models in Paper I never accrete more than $2.5 \times 10^{50} \mathrm{erg} \mathrm{s}$, implying that $T / W=0.14$ might hardly be achievable. We conclude that the bar-mode instability may be irrelevant in the SN Ia context, and only the $r$-mode instability may be relevant in accreting white dwarfs.

Lindblom (1999) derived the time scale for the growth of $r$-mode instability in rigidly rotating stars as $1 / \tau_{\mathrm{r}}=$ $K \int \rho(r \Omega)^{6} \mathrm{~d} r$, where $K=(2 \pi / 25)(4 / 3)^{8} G / c^{7}$. Using the mean values for density, radius and angular velocity $(\bar{\rho}, \bar{R}$ and $\bar{\Omega})$, this time scale can be given as $1 / \tau_{\mathrm{r}} \approx K \bar{\rho} \bar{\Omega} \bar{R}^{7} / 7=(3 K / 4 \pi) M \bar{\Omega}^{6} \bar{R}^{4}$. Since we have $\bar{\Omega}=J /\left(k M \bar{R}^{2}\right), T=(k / 2) M \bar{\Omega}^{2} \bar{R}^{2}$ and $W=$ $\gamma G M^{2} / \bar{R}$, where $k$ and $\gamma$ vary according to the stellar structure, the time scale $\tau_{\mathrm{r}}$ can be expressed in terms of $M, J$ and $T / W$ as the following:

$$
\begin{aligned}
\log _{10} \tau_{\mathrm{r}}[\mathrm{yr}]= & -3.94+10 \log _{10}\left(J / 10^{50}\right)-19 \log _{10}\left(M / M_{\odot}\right) \\
& -8 \log (T / W)-8 \log _{10} \gamma-2 \log _{10} k
\end{aligned}
$$

In our white dwarf models, $k$ and $\gamma$ are in the range of $0.1 \sim$ 0.14 and $1.0 \sim 1.6$, respectively.

In Fig. 11, lines of constant $\tau_{\mathrm{r}}$ are plotted in the $M-J$ plane, in the range of $1.4<M / M_{\odot}<1.9$ and $0.5<$ $J /\left[10^{50} \mathrm{erg} \mathrm{s}\right]<2.0$. Here, the $M-J$ and $M-T / W$ relations given by Eqs. (19) and (29) are used, with the fixed values of $\gamma=1.0$ and $k=0.14$. Values of $\tau_{\mathrm{r}}$ are found to be about $\sim 10^{3} \ldots \sim 10^{5} \mathrm{yr}$ in the considered range of $M$ and $J$. This estimate indicates that the $r$-mode instability might have enough time to operate in white dwarfs during the accretion phase, if $\dot{M}=\sim 10^{-7} M_{\odot} \mathrm{yr}^{-1}$, which is typical for the Single Degenerate scenario for SN Ia progenitors.

\subsection{Evolutionary scenarios}

Let us define the accretion time scale as $\tau_{\text {acc }}$, and the time scale for the loss of angular momentum due to $r$-mode instability as $\tau_{\mathrm{J}}$, respectively.

\subsubsection{Case $\mathrm{A}: \tau_{\mathrm{J}}<\tau_{\mathrm{acc}}$}

When a white dwarf accretes sufficient mass and angular momentum, the $r$-mode instability begins to operate, and as a consequence the white dwarf loses angular momentum. Here we assume that, even though the angular momentum loss rate is small initially, it will increase during the white dwarf spin-up, to the extent that $\tau_{\mathrm{J}}<\tau_{\text {acc }}$, i.e., that the loss of angular momentum occurs more rapidly than its accretion. Such a scenario is motivated by the r-mode angular momentum loss time scale as given by Eq. (36).

In this case, the growth of the white dwarf angular momentum - and thereby the growth of its mass - is limited by the angular momentum loss; it ignites carbon at the center when $J$ approaches $J_{\mathrm{SNIa}}$, as illustrated by Path A1 in Fig. 11. The end point of Path A1, which defines the explosion mass $M_{\mathrm{A} 1}$, depends on both the angular momentum loss time scale $\tau_{\mathrm{r}}$ and the 


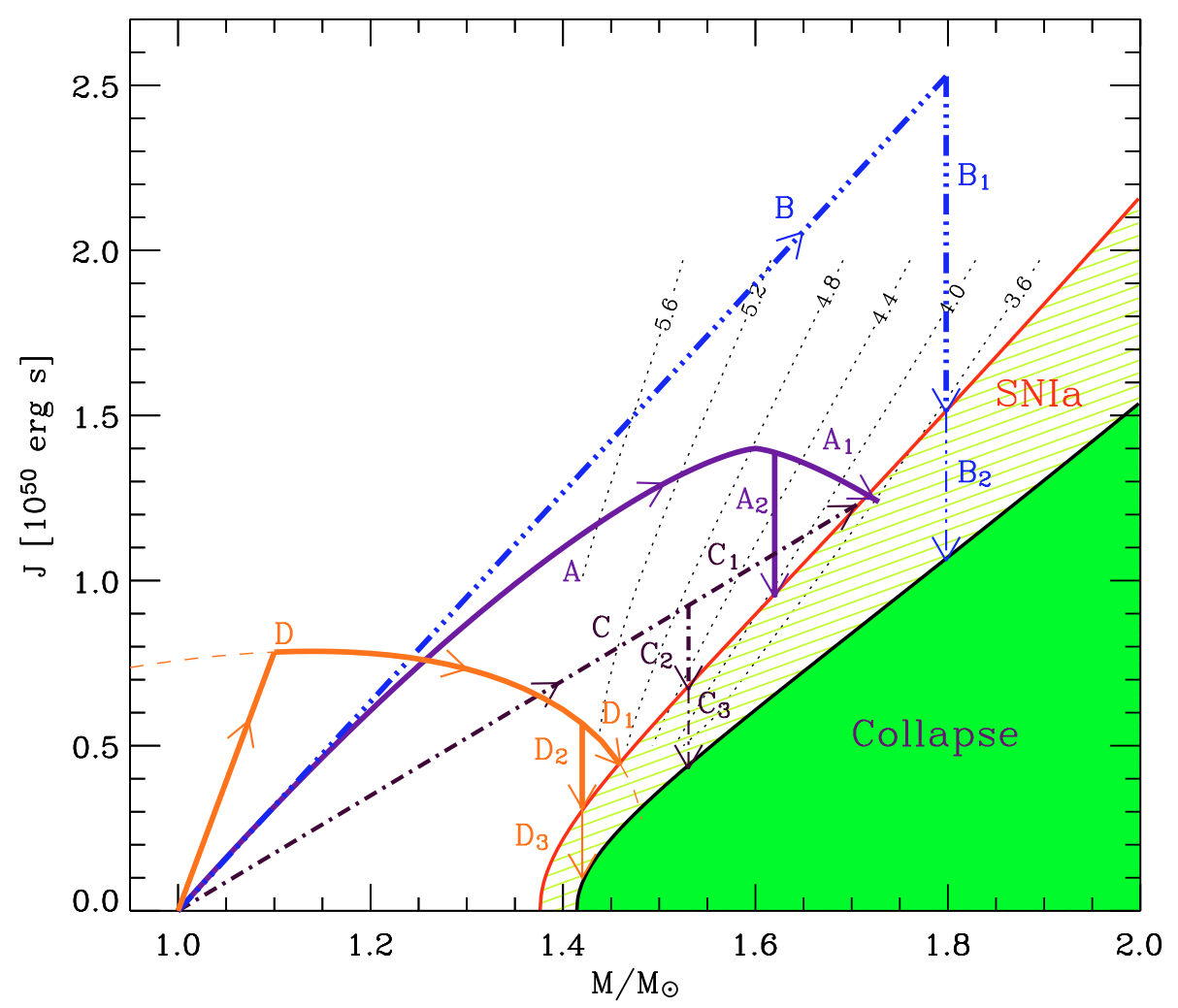

Fig. 11. Schematic supernova progenitor evolution scenarios in the $M-J$ plane. Thick lines starting at a white dwarf mass of $1 M_{\odot}$ with $J=0$ indicate various possible evolutionary paths of accreting white dwarfs: with angular momentum accretion and simultaneous angular momentum loss (A), without angular momentum loss during the accretion phase (B), with inefficient angular momentum accretion (C), and with maximum internal angular momentum transport efficiency (D); (see text for details). The region where $J_{\mathrm{EC}, \mathrm{CO}} \lesssim J \lesssim J_{\mathrm{SNIa}}$ is hatched, and the region where $J \lesssim J_{\mathrm{EC}, \mathrm{CO}}$ is colored. For $\dot{M} \simeq 10^{-7} \ldots 10^{-6} \mathrm{M} \mathrm{yr}^{-1}$, a supernova explosion is expected at the upper borderline of the hatched area, $J=J_{\mathrm{SNIa}}$; cf. Eq. (23). The thin dashed line gives the $M-J$ relation of rigidly rotating white dwarfs at critical rotation. Dotted lines denote lines of constant growth time of the $r$-mode instability ( $\tau_{\mathrm{r}}$; Eq. (36)) and are labeled with the logarithm of the growth time in years.

angular momentum accretion efficiency. One dimensional evolutionary calculations with an angular momentum accretion efficiency as in Paper I show that $\tau_{\mathrm{J}} \simeq \tau_{\mathrm{r}}$ results in $M_{\mathrm{A} 1} \simeq 1.6 M_{\odot}$ and $1.5 M_{\odot}$, for the initial masses of $0.8 M_{\odot}$ and $1.0 M_{\odot}$, respectively (Knaap et al., in preparation).

In some binary systems, the mass budget may be limited and the mass transfer stops before the white dwarf reaches $M_{\mathrm{Al}}$, but at a mass higher than the classical Chandrasekhar mass. Then, the white dwarf evolves vertically downward in the $M-J$ plane as illustrated by path A2. Since $\tau_{\mathrm{J}} \lesssim 10^{6} \mathrm{yr}$ in this case, the central temperature at the moment when the white dwarf reaches $J_{\text {SNIa }}$ is high enough $\left(T \simeq 10^{8} \mathrm{~K}\right)$ to ignite carbon at $\rho_{\mathrm{c}} \simeq 2 \times 10^{9} \mathrm{~g} \mathrm{~cm}^{-3}$ (see Paper I). Therefore, both Path A1 and Path A2 lead to the production of SNe Ia. The masses of exploding white dwarfs through Path A are in the range of $1.38 M_{\odot} \lesssim M \lesssim M_{\mathrm{A} 1}$. Electron-capture induced collapse cannot occur in Case A.

\subsubsection{Case B: $\tau_{\mathrm{J}}>\tau_{\mathrm{acc}}$}

If $\tau_{\mathrm{J}}>\tau_{\text {acc }}$, the evolutionary path of the accreting white dwarf will critically depend on the angular momentum accretion efficiency. If it is so high that the white dwarf angular momentum always exceeds $J_{\text {SNIa }}$ during the accretion phase, the white dwarf mass will continue to grow until the mass transfer comes to an end. Once the mass transfer stops, carbon ignition will be delayed until the white dwarf loses enough angular momentum (Path B in Fig. 11). Its final fate will depend on the angular momentum loss time scale. If it is larger than the white dwarf cooling time (i.e., $\tau_{\mathrm{J}} \gtrsim 10^{9} \mathrm{yr}$ ), carbon ignition will occur after the white dwarf core has crystallized - i.e., when $J \simeq J_{\mathrm{EC}, \mathrm{CO}}-$, which leads to electron-capture induced collapse and results in a neutron star (Nomoto \& Kondo 1991). This case is illustrated by Path B2.

If $\tau_{\mathrm{J}}$ is short enough for carbon to ignite in the liquid state in the white dwarf core, a SN Ia explosion will occur (Path B1). The mass of exploding white dwarfs can vary from $1.38 M_{\odot}$ to $2.0 M_{\odot}$, depending on the mass budget in the binary system. However, in this case, the large angular momentum loss time scale of more than $10^{6} \mathrm{yr}$ leads to an ignition density $\left(\rho_{\text {ign }}\right)$ significantly above $\sim 2 \times 10^{9} \mathrm{~g} \mathrm{~cm}^{-3}$. As SNe Ia at such high densities produce highly non-solar neutron-rich iron group element ratios (Iwamoto et al. 1999), this scenario cannot be considered as the major evolutionary path. 


\subsubsection{Case C: $\tau_{\mathrm{J}}>\tau_{\mathrm{acc}}$ and low $\dot{J}_{\mathrm{acc}}$-efficiency}

If the angular momentum accretion efficiency is sufficiently low, white dwarfs can reach $J_{\mathrm{SNI}}$ to produce a $\mathrm{SN}$ Ia during the accretion phase even if $\tau_{\mathrm{J}}>\tau_{\mathrm{acc}}$, as long as enough mass is supplied by the binary companion, as illustrated by Path $\mathrm{C} 1$. If the mass transfer stops when $M \gtrsim 1.38 M_{\odot}$ but before reaching $J_{\text {SNIa }}$, carbon ignition will be delayed, and its outcome will depend on the time scale for angular momentum loss, as in the Case B. If $\tau_{J}$ is shorter than the cooling time scale, a SN Ia explosion is expected soon after $J$ reaches $J_{\text {SNIa }}$ (Path C2). Otherwise, carbon burning will occur only when $J \simeq J_{\mathrm{EC}, \mathrm{CO}}$ and result in electron-capture induced collapse to form a neutron star (Path C3). The masses of exploding white dwarfs through Path $\mathrm{C}$ are between $1.38 M_{\odot}$ and the end point of Path $\mathrm{C} 1\left(M_{\mathrm{C} 1}\right)$.

\subsubsection{Case D: Extreme J-transport efficiency}

Although Paper I showed that non-magnetic accreting white dwarfs are expected to rotate differentially, the existence of rigidly rotating white dwarfs, through extremely efficient transport of angular momentum by magnetic torques, cannot be excluded. If this is the case, the white dwarf surface begins to rotate critically soon after the onset of mass accretion, as shown by previous work (Piersanti et al. 2003a; Uenishi et al. 2003; Saio \& Nomoto 2004)

The angular momentum of a rigidly rotating white dwarf at critical rotation has an absolute maximum at about $1.13 M_{\odot}$, and decreases as the mass increases further (see Fig. 10). Therefore, once a rigidly rotating white dwarf reaches critical rotation, it must lose angular momentum during the accretion when $M \gtrsim 1.13 M_{\odot}$. The $r$-mode instability could remove angular momentum, if $\tau_{\mathrm{J}}<\tau_{\mathrm{acc}}$. However, in our rigidly rotating white dwarf models at critical rotation, the estimated growth time scale for the $r$-modes, $\tau_{\mathrm{r}}$, becomes smaller than $10^{6} \mathrm{yr}$ only when $M \gtrsim 1.25 M_{\odot}$. It is therefore questionable whether the $r$-mode instability can be an efficient mechanism to remove angular momentum during the evolution of rigidly rotating white dwarfs. As mentioned earlier, the bar-mode instability also cannot operate as the high required value of $T / W \simeq 0.14$ cannot be reached in rigidly rotating white dwarfs. Uenishi et al. (2003) and Saio \& Nomoto (2004) considered the viscous transport of angular momentum from the white dwarf into the accretion disk, so that a rigidly rotating white dwarf with $M \gtrsim 1.13 M_{\odot}$ may keep rotating critically with continuous accretion of mass, while angular momentum decreases progressively.

Within this picture, the evolution of rigidly rotating white dwarfs will follow Path D in Fig. 11. If the binary companion supplies sufficient mass to reach $M \simeq 1.46 M_{\odot}$, a SN Ia explosion occurs during the accretion phase (Path D1). If mass transfer ceases before reaching $M \simeq 1.46 M_{\odot}$ but after $M \gtrsim$ $1.38 M_{\odot}$, carbon ignition at the center will be delayed until enough angular momentum is lost (Paths D2 and D3), with the fate of the white dwarf depending on $\tau_{J}$, as in the case B. Exploding white dwarfs through Path $\mathrm{D}$ have masses in the range $1.38 \lesssim M / M_{\odot} \lesssim 1.46$ (Uenishi et al. 2003).

\subsubsection{Diversity in $M_{\mathrm{SNla}}$}

The scenarios outlined above show that the masses of exploding white dwarfs $\left(M_{\mathrm{SNIa}}\right)$ may not be uniform, but are expected to be diverse, with an explosion mass range depending on the evolutionary path realized by nature. The minimum mass is the canonical value of $1.38 M_{\odot}$, while the maximum possible mass $\left(M_{\max }\right)$ remains uncertain; Paths B and D result in the largest $\left(1.38 \lesssim M_{\mathrm{SNIa}} \lesssim 2.0 M_{\odot}\right)$ and smallest $\left(1.38 \lesssim M_{\text {SNIa }} \lesssim 1.46 M_{\odot}\right)$ diversity in mass, respectively. Paths A and $\mathrm{C}$ may lead to $M_{\max }<2.0 M_{\odot}$, with the exact upper mass limit depending on the angular momentum loss time scale and the angular momentum accretion efficiency.

\section{Observable consequences of super-Chandrasekhar mass explosions}

Recent results of 3D numerical simulations of SN Ia explosion indicate that the Delayed Detonation scenario can explain the major features of observed SNe Ia, in particular the explosion energy and nucleosynthesis (Gamezo et al. 2004). In this scenario, the explosion starts out as a deflagration, with a deflagration-to-detonation transition (DDT) occurring during the explosion. However, so far the DDT does not occur selfconsistently in the explosion models but needs to be put in by hand. Lisewski et al. (2000) showed that a DDT in a SN Ia may occur if turbulent motions with velocities of $\sim 10^{8} \mathrm{~cm} \mathrm{~s}^{-1}$ occur on scales of $\approx 10^{6} \mathrm{~cm}$. Such high velocities are not expected for Rayleigh-Taylor-driven turbulence in SN Ia deflagration models. The rotational velocities in our white dwarf models are typically from a few to several times $10^{8} \mathrm{~cm} \mathrm{~s}^{-1}$ (Fig. 12). Therefore, even if the white dwarf is spun down by the expansion during the explosion, rotation could provide enough kinetic energy to enforce the turbulence to the level required for a DDT (see also Paper I).

If the occurrence of a DDT is indeed related to the strength of rotation, the DDT might occur earlier (i.e., at higher transition densities) in more massive white dwarfs, as those rotate more rapidly (cf. Fig. 12). More massive white dwarfs will produce more ${ }^{56} \mathrm{Ni}$ in such a case, since $1 \mathrm{D}$ models show that a higher transition density results in a more energetic explosion and thus in the production of more ${ }^{56} \mathrm{Ni}$ (e.g. Höflich \& Khokhlov 1996). Even for a pure deflagration, more massive white dwarfs are likely to yield more ${ }^{56} \mathrm{Ni}$, since they can provide more fuel for the nuclear burning. Therefore, we may expect the production of more ${ }^{56} \mathrm{Ni}$, and thus brighter $\mathrm{SNe} \mathrm{Ia}$ (Arnett 1982), from more massive white dwarfs.

The production of more ${ }^{56} \mathrm{Ni}$ in more massive white dwarfs is also required for our super-Chandrasekhar mass scenario to satisfy the observational constraints on the kinetic energy $\left(E_{\mathrm{kin}}\right)$ of SN Ia. Observed ejecta velocities imply $E_{\text {kin }} \approx 10^{51} \mathrm{erg}$, assuming that SNe Ia come from Chandrasekhar mass white dwarfs (e.g. Branch 1992). In other words, SNe Ia have a specific kinetic energy $\left(e_{\text {kin }}\right)$ of about $3.6 \times 10^{17} \mathrm{erg} \mathrm{g}^{-1}$. No clear correlation between SN Ia peak brightness and ejecta velocity seems to exist (e.g. Hatano et al. 2000), which might imply that SNe Ia have a similar specific kinetic energy on average. A diversity in the mass of exploding white dwarfs might 


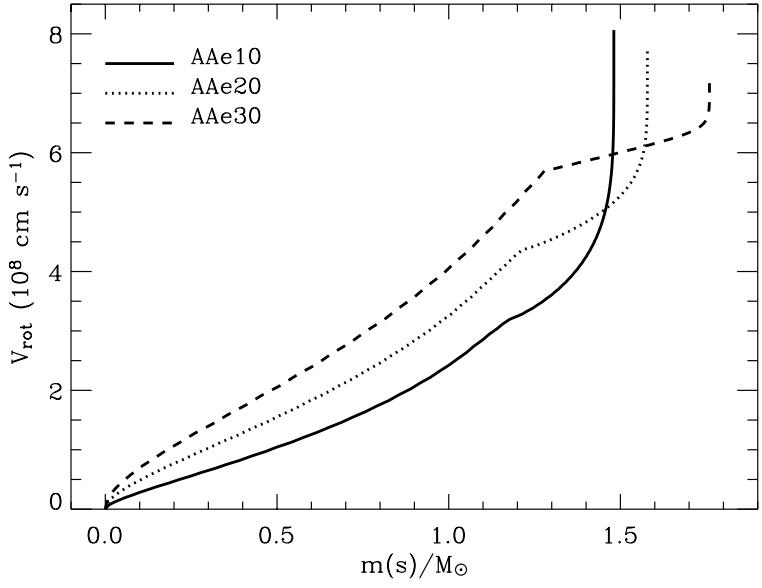

Fig. 12. Equatorial rotational velocity as a function of the mass coordinate in the pre-supernova models AAe10, AAe20 and AAe30.

thus imply that more massive white dwarfs yield, in general, more specific nuclear energy $\left(e_{\mathrm{n}}\right)$ in the explosion, since the specific binding energy $\left(e_{\mathrm{BE}}\right)$ increases with increasing mass (Fig. 9). Although 1D explosion models show no strict correlation between $e_{\mathrm{n}}$ and the produced mass of ${ }^{56} \mathrm{Ni}\left(M_{\mathrm{Ni}}\right)$, there is certainly a general tendency that a higher $e_{\mathrm{n}}$ corresponds to more $M_{\mathrm{Ni}}$ (e.g. Branch 1992). Therefore, larger nickel masses are expected, on average, in more massive white dwarfs to explain the observed SN Ia kinetic energies.

Figure 13a shows the required nuclear energy $E_{\mathrm{n}}$ to give $e_{\text {kin }}=3.6 \ldots 5.4 \times 10^{17} \mathrm{erg} \mathrm{g}^{-1}$ as a function of the white dwarf mass, where Eq. (32) is used for the binding energy of white dwarfs at $\rho_{\mathrm{c}}=2 \times 10^{9} \mathrm{~g} \mathrm{~cm}^{-3}$. Here, the adopted range of $e_{\text {kin }}$ corresponds to what is typically assumed in the literature (e.g. Gamezo et al. 2004). The variation of $E_{\mathrm{n}}$ in this figure remains within $75 \%$ in the considered mass range. 1D Chandrasekhar mass explosion models by Höelfich $\&$ Khokhlov (1996) show that $e_{\text {kin }}=3.6 \ldots 5.4 \times 10^{17} \mathrm{erg} \mathrm{g}^{-1}$ can be achieved with $M_{\mathrm{Ni}} \approx 0.2 \ldots 0.8$, within the Delayed Detonation scenario. Assuming the average $M_{\mathrm{Ni}}$ is proportional to $E_{\mathrm{n}}$, we can estimate a possible range of $M_{\mathrm{Ni}}$ which will yield $e_{\text {kin }}=3.6 \ldots 5.4 \times 10^{17} \mathrm{erg} \mathrm{g}^{-1}$, as in Fig. 13b. Although the estimated range of $M_{\mathrm{Ni}}$ is very uncertain and needs to be confirmed by detailed explosion models, this consideration leads us to conclude that rotation has the potential to plausibly explain the observed diversity of SN Ia peak brightnesses: the estimated range of $M_{\mathrm{Ni}}$ covers most of the observed range of $0.1 M_{\odot} \lesssim M_{\mathrm{Ni}} \lesssim 1.14 M_{\odot}$, where the lower and upper limits refer to SN 1991bg and SN 1991T, respectively (Leibundgut 2000).

During the last years, differences in the chemical composition - notably, in the $\mathrm{C} / \mathrm{O}$ ratio or in the overall metallicity of exploding white dwarfs have been invoked to explain the occurrence of different nickel masses for Chandrasekhar-mass white dwarf explosions (Umeda et al. 1999; Höflich et al. 2000; Timmes et al. 2003). However, recent 3D simulations by Röpke $\&$ Hillebrandt (2004) indicate that different $\mathrm{C} / \mathrm{O}$ ratios have a negligible effect on $M_{\mathrm{Ni}}$. Also the metallicity dependence appears not able to explain the full observed spread (Timmes et al. 2003).
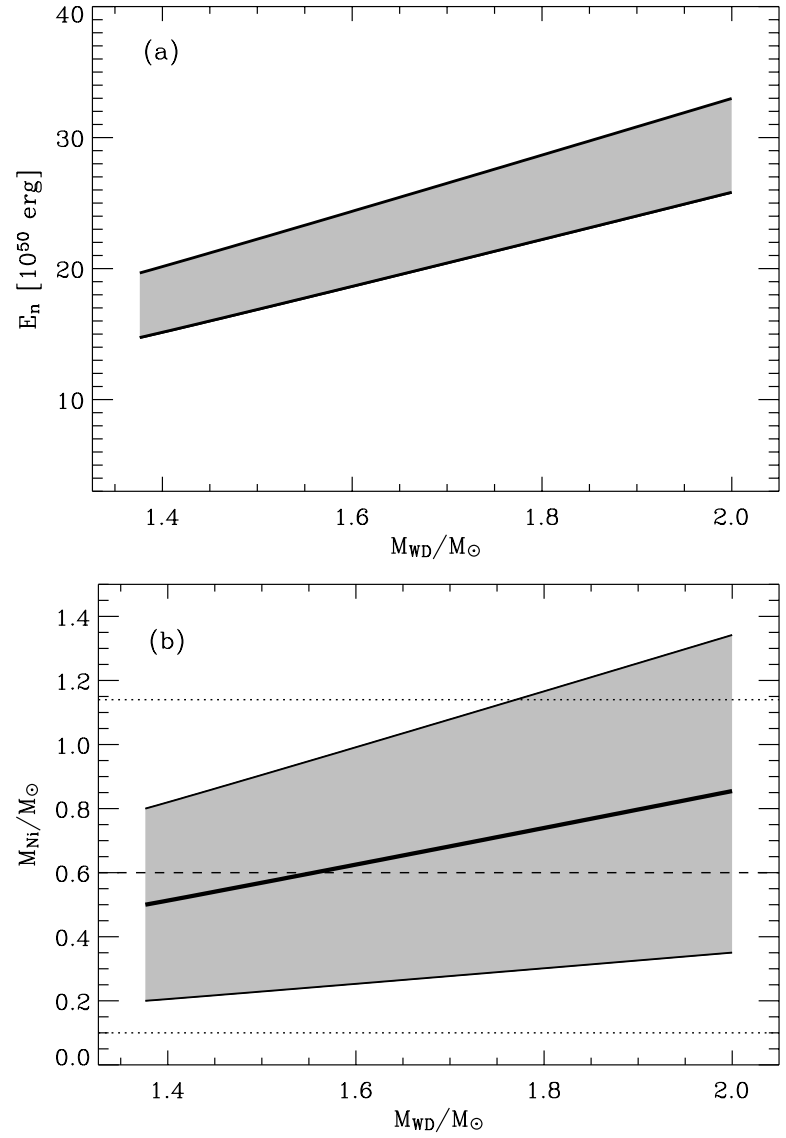

Fig. 13. a) Nuclear energy required to explain the specific kinetic energy $\left(e_{\text {kin }}\right)$ of observed SNe Ia, as a function of the white dwarf mass, i.e, $E_{\mathrm{n}}=E_{\mathrm{BE}}+M_{\mathrm{WD}} e_{\mathrm{kin}}$. The binding energy given by Eq. (32) is used here. Lower and upper bounds correspond to $e_{\mathrm{kin}}=3.6 \times 10^{17} \mathrm{erg} \mathrm{g}^{-1}$ and $5.4 \times 10^{17} \mathrm{erg} \mathrm{g}^{-1}$, respectively. b) Expected nickel mass produced in a SN Ia explosion as a function of the white dwarf mass, assuming that $M_{\mathrm{Ni}}$ is proportional to $E_{\mathrm{n}}$. Lower and upper limits are computed from the assumption that minimum and maximum values for $M_{\mathrm{Ni}}$ at $M=1.38 M_{\odot}$ are $0.2 M_{\odot}$ and $0.8 M_{\odot}$, respectively. The thick solid line in the middle of the gray-colored region defines the mean value between these two limits. The dashed line gives the mean value of $M_{\mathrm{Ni}}$ in observed SNe Ia. Lower and upper dotted lines indicate the observationally estimated minimum and maximum $M_{\mathrm{Ni}}$, respectively (Leibundgut 2000; see text).

Another possible cause for the supernova brightness diversity within the Chandrasekhar mass scenario might be related to differences in the carbon ignition density or in the transition density for the DDT. However, for any evolutionary path toward the Chandrasekhar limit, the accretion rates are restricted to $10^{-7} \ldots 10^{-6} M_{\odot} \mathrm{yr}^{-1}$, since otherwise a white dwarf cannot efficiently grow in mass. Since the thermal evolution of non-rotation accreting white dwarfs is mainly determined by the accretion rate, one cannot expect a significant diversity in the ignition conditions in Chandrasekhar mass white dwarfs. Furthermore, none of these possibilities could explain the large amount of $M_{\mathrm{Ni}} \approx 1.0 M_{\odot}$ in SN $1991 \mathrm{~T}$ (Fisher et al. 1999). This leaves a diversity of masses above the classical Chandrasekhar mass, caused by rotation, as a promising explanation for the origin of the diversity in the SN Ia brightness. 
Also the diversity in the ejecta velocity of SNe Ia, which was attributed to different explosion mechanisms by Hatano et al. (2000), can be related to different white dwarf progenitor masses. Given that $E_{\mathrm{kin}}=E_{\mathrm{BE}}-E_{\mathrm{n}}$, SNe Ia with the same brightness may have somewhat different amounts of kinetic energy, if two different masses result in similar nuclear energy and $M_{\mathrm{Ni}}$. Similarly, it is possible that some very bright SNe Ia have a smaller kinetic energy than less bright ones, depending on how much nuclear energy is released at different masses. Therefore, in our scenario, the kinetic energy in SNe Ia may not necessarily correlate with the brightness, in accordance with the observations.

The possible correlation between progenitor mass $M_{\mathrm{SNI}}$ and the SNe Ia brightness ( $M_{\mathrm{SNIa}}-L_{\mathrm{SNIa}}$ correlation) could be tested in various ways. Observations indicate that more than $70 \%$ of SNe Ia are fairly homogeneous, giving similar brightnesses $\left(M_{\mathrm{Ni}} \simeq 0.6 M_{\odot}\right)$ and light-curves, while about $20 \% \ldots 30 \%$ are SN 199bg-type (sub-luminous) or SN 1991T-type (over-luminous) (Hillebrandt \& Niemeyer 2000; Leibundgut 2000). If the $M_{\mathrm{SNIa}}-L_{\mathrm{SNIa}}$ correlation were to explain the observed peak brightness variation, the distribution of progenitor masses $\left(P_{M_{\mathrm{SNI}}}\right)$ should not be flat, but peak at a certain mass which is to represent the normal SNe Ia. Explosions from lower and higher masses may then relate to sub-luminous and over-luminous SNe Ia, respectively. Population synthesis studies are needed to investigate whether this scenario may result in a progenitor mass distribution which can be consistent with the observed distribution of nickel masses.

It is more straightforward to relate the $M_{\mathrm{SNIa}}-L_{\mathrm{SNIa}}$ correlation to the fact that $\mathrm{SNe}$ Ia from early type galaxies are systematically less luminous than those from late type galaxies (e.g. Ivanov et al. 2000). Since SN Ia progenitors must be, in general, much older in early type galaxies than in late type ones, SN Ia progenitor systems in early type galaxies should have a systematically smaller mass budget, which results in smaller $M_{\text {SNIa }}$ on average, and thus in less luminous SNe Ia. Similarly, if the progenitor mass distribution changes systematically as a function of the metallicity of the progenitor systems, SNe Ia luminosities will also change systematically according to cosmic age. For instance, if the initial mass distribution of white dwarfs does not sensitively depend on metallicities, we expect the peak of the progenitor mass distribution at a lower mass for lower metallicity (Langer et al. 2000). We thus expect dimmer SNe Ia for lower metallicity, which may have important consequences for the cosmological interpretation of supernova distances at high redshift.

The aspherical nature of rapidly rotating white dwarfs might, in principle, result in an observable polarization, as found in SN 1999by (Howell et al. 2001) and SN 2001el (Wang et al. 2003). However, the white dwarf rotation per se may not be able to create an aspherical shape of the ejecta: Since white dwarf models with $\rho_{\mathrm{c}}=2 \times 10^{9} \mathrm{~g} \mathrm{~cm}^{-3}$ have a specific angular momentum of $\sim 10^{16} \mathrm{~cm}^{2} \mathrm{~s}^{-1}$ (Fig. 6), the rotational velocity of the ejecta will be $V_{\text {ejecta,rot }} \simeq j / R_{\text {ejecta }} \simeq$ $0.43\left[\mathrm{~km} \mathrm{~s}^{-1}\right]\left(j / 3 \times 10^{16} \mathrm{~cm}^{2} \mathrm{~s}^{-1}\right) /\left(R_{\text {ejecta }} / 10 R_{\odot}\right)$, which is negligible compared to the observed ejecta velocities in radial direction $\left(\sim 10^{4} \mathrm{~km} \mathrm{~s}^{-1}\right)$. Instead, an aspherical distribution of the nuclear fuel in rotating white dwarfs (see Fig. 2) and a consequent aspherical explosion could be more important in producing non-radial movements in the ejecta. Future 3D explosion models are needed to relate such effects to our rotating pre-supernova models.

\section{Electron-capture induced collapse}

Although the present study focuses on Single Degenerate $\mathrm{SNe}$ Ia progenitors, our 2D models may also facilitate the study of double (CO-CO and $\mathrm{CO}-\mathrm{ONeMg}$ ) white dwarf mergers. During such mergers, dynamically unstable mass transfer will result in the formation of a thick accretion disk around the more massive white dwarf (e.g. Benz et al. 1990). While it is uncertain how the mass accretion from the thick disk onto the white dwarf proceeds, a rapidly spinning super-Chandrasekhar mass merger product can be expected, as recently shown by Piersanti et al. (2003b). In this study, however, the assumption of rigid rotation again strongly restricts the possible explosion masses. Furthermore, the estimated ratio of rotational to gravitational energy of $\sim 0.14$ in their $1 \mathrm{D}$ rapidly rotating white dwarf models exceeds by far the maximum possible value for such configurations (Ostriker \& Tassoul 1968; see also Sect. 6.4.4), casting doubt on the general validity of their results of the reduced and self-regulated accretion rates due to rotation.

Saio \& Nomoto (2004) recently suggested that a double CO-white dwarf merger leads to accretion at rates near the Eddington limit $\left(\sim 10^{-5} M_{\odot} \mathrm{yr}^{-1}\right)$, which, after off-center carbon ignition, will produce a rapidly spinning ONeMg white dwarf. When the central density in such an $\mathrm{ONeMg}$ white dwarf reaches about $4 \times 10^{9} \mathrm{~g} \mathrm{~cm}^{-3}$, electron capture on ${ }^{24} \mathrm{Mg}$ and ${ }^{20} \mathrm{Ne}$ begins, and the collapse of the star to form a neutron star is the expected outcome (Nomoto \& Kondo 1991). Strongly magnetized neutron stars with $B \simeq 10^{15} \mathrm{G}$ and $P \simeq 1 \mathrm{~ms}$ produced by such events are proposed as the central engine of cosmic gamma-ray bursts (e.g. Dar et al. 1992; Usov 1992; Duncan \& Thomson 1992; Yi \& Blackman 1998; Ruderman et al. 2000). While non-rotating ONeMg white dwarfs undergo electron-capture (EC) induced collapse when their mass reaches the Chandrasekhar-limit, rotating $\mathrm{ONeMg}$ white dwarfs may need a super-Chandrasekhar mass for such collapse. Since a single ONeMg white dwarf may be as massive a $1.35 M_{\odot}$, the formation of $\mathrm{ONeMg}$ white dwarfs as massive as $\sim 2.7 M_{\odot}$ by mergers is, in principle, possible.

Notably, as shown in Fig. 6, the specific angular momentum in pre-collapse white dwarfs with $M_{\mathrm{EC}, \mathrm{ONM}} \gtrsim 1.5 M_{\odot}$ is larger than $j \approx 1.5 \times 10^{16} \mathrm{~cm}^{2} \mathrm{~s}^{-1}$, which is the maximum angular momentum of a rigidly rotating neutron star. Therefore, either a large amount of angular momentum must be lost during the collapse, or the resulting neutron stars have to rotate differentially. In the latter case, the expected mean spin rate ranges from a few to several times $\sim 10^{4} \mathrm{~s}^{-1}$.

According to our evolutionary scenarios of accreting $\mathrm{CO}$ white dwarfs, EC induced collapse is also expected to occur if carbon ignition at the center of a super-Chandrasekhar mass CO white dwarf is delayed for a long time (Paths B2, 
C3 and D3; see Fig. 11). Figure 6 shows that the specific angular momentum in pre-collapse $\mathrm{CO}$ white dwarfs $\left(\rho_{\mathrm{c}}=\right.$ $10^{10} \mathrm{~g} \mathrm{~cm}^{-3}$ ), ranges from $10^{16} \mathrm{~cm}^{2} \mathrm{~s}^{-1}$ to $4 \times 10^{16} \mathrm{~cm}^{2} \mathrm{~s}^{-1}$ where $1.5 \lessgtr M / M_{\odot} \lesssim 2.0$. This is again sufficient for the formation of differentially rotating neutron stars of with spin rates of $\sim 10^{4} \mathrm{~s}^{-1}$.

In both cases, such rapidly rotating, newly formed neutron stars are believed to lose angular momentum via the $r$-mode instability, magnetic dipole radiation, and/or magnetic torques. More importantly, if the differential rotation could amplify the magnetic field up to $10^{15} \mathrm{G}$ in such newly born neutron stars (Ruderman et al. 2000), or such fields were formed by a dynamo process right after the collapse (Duncan \& Thomson 1992), the necessary conditions to form a cosmic GRBs due to electro-magnetic beaming - i.e., $\Omega \approx 10^{4} \mathrm{~s}^{-1}$ and $B \approx 10^{15} \mathrm{G}-$ might be satisfied (cf. Wheeler et al. 2000; Zhang \& Mészáros 2001). Although it is a matter of debate whether this channel could be a major source for GRBs (e.g. Fryer et al. 1999; Middleditch 2004), its possibility deserves careful investigation. Our 2D models might provide an ideal starting point for such a study.

\section{Conclusion}

We present two-dimensional models of differentially rotating massive white dwarfs, with internal rotation laws that are derived from evolutionary models of accreting white dwarfs (Paper I). In these models, the shear rate inside the white dwarf is limited by the threshold value for the onset of the dynamical shear instability. We find that various physical quantities of these 2D white dwarf models - such as angular momentum and binding energy - can be accurately expressed as analytical functions of the white dwarf mass and central density, and do not sensitively depend on the details of the rotation profile (Sect. 4). In particular, we construct models with $\rho_{\mathrm{c}}=2 \times$ $10^{9} \mathrm{~g} \mathrm{~cm}^{-3}$ and $10^{10} \mathrm{~g} \mathrm{~cm}^{-3}$, which represent the pre-explosion and pre-collapse stages of $\mathrm{CO}$ white dwarfs, respectively, and models with $\rho_{\mathrm{c}}=4 \times 10^{9} \mathrm{~g} \mathrm{~cm}^{-3}$ which correspond to the pre-collapse stage of ONeMg white dwarfs. Based on these results, in particular using the mass-angular momentum relation of our 2D pre-supernova models, we present new scenarios for the evolution of SNe Ia progenitors (Sect. 6.4; Fig. 11), in the frame of a new paradigm of SN Ia explosion at a variety of super-Chandrasekhar masses. By making use of the binding energies of our pre-supernova white dwarf models, we suggest that the SN Ia brightness may be proportional to the exploding white dwarf mass on average, and that this correlation could explain various aspects of the SNe Ia diversity (Sect. 7). We also briefly discussed the electron-capture induced collapse of rapidly rotating super-Chandrasekhar mass white dwarf as a potential candidate for the central engine of a sub-class of cosmic gamma-ray bursts (Sect. 8). In conclusion, by providing 2D white dwarf models at the pre-explosion and pre-collapse stages (Sect. 3), together with analytic relations describing their global properties (Sect. 4), we hope to facilitate future studies of such objects, including investigations on the role of the $r$-mode instability and the mass and angular momentum accretion efficiency for the evolution of accreting white dwarfs, population synthesis models of the distribution of white dwarf supernova progenitor masses, its relation to SNe Ia diversity, and most importantly, multi-dimensional simulations of SN Ia explosion and electron-capture induced collapse of rotating white dwarfs.

Acknowledgements. This research has been supported in part by the Netherlands Organization for Scientific Research (NWO).

\section{References}

Arnett, W. D. 1982, ApJ, 253, 785

Benz, W., Cameron, A. G. W., Press, W., \& Bowers, R. L. 1990, ApJ, 348,647

Blinnikov, S. I., Dunina-Barkovskaya, N. G., \& Nadyozhin, D. K. 1996, ApJS, 106, 171

Branch, D. 1992, ApJ, 392, 35

Cassisi, S., Iben, I., Jr., \& Tornambé, A. 1998, ApJ, 496, 376

Dar, A., Kozlovsky, B., Nussinov, S., \& Ramaty, R. 1992, ApJ, 388, 164

Duncan, R. C., \& Thompson, C. 1992, ApJ, 392, L9

Durisen, R. H. 1977, ApJ, 213, 145

Durisen, R. H., \& Imamura, J. N. 1981, ApJ, 243, 612

Fisher, A., Branch, D., Hatano, K., \& Baron, E. 1999, MNRAS, 304, 67

Fryer, C., Benz, W., Herant, M., \& Colgate, S. A. 1999, ApJ, 516, 892

Gamezo, V. N., Khokhlov, A. M., \& Oran, E. S. 2004, Phys. Rev. L., 92, 211102-1

Hachisu, I. 1986, ApJS, 61, 479

Hachisu, I., Kato, M., \& Nomoto, K. 1996, ApJ, 470, L97

Han, Z., \& Podsiadlowski, Ph. 2004, MNRAS, 350, 1301

Hatano, K., Branch, D., \& Lentz, E. J., et al. 2000, ApJ, 543, L49

Heger, A., \& Langer, N. 2000, ApJ, 544, 1016

Heger, A., Woosley, S. E., \& Spruit, H. C. 2004, ApJ, submitted [arXiv: astro-ph/0409422]

Hillebrandt, W., \& Niemeyer, J. C. 2000, ARA\&A, 38, 191

Hirschi, R., Meynet, G., \& Maeder, A. 2004, A\&A, 425, 649

Höflich, P., \& Khokhlov, A. M. 1996, ApJ, 457, 500

Höflich, P., Nomoto, K., Umeda, H., \& Wheeler, J. C. 2000, ApJ, 528, 590

Howell, D. A., Höflich, P., Wang, L., \& Wheeler, J. C. 2001, ApJ, 556, 302

Ivanov, V. D., Hamuy, M., \& Pinto, Ph. A. 2000, ApJ, 542, 588

Iwamoto, K., Brachwitz, F., \& Nomoto, K., et al. 1999, ApJS, 125, 439

Kato, M., \& Hachisu, I. 2004, ApJ, 613, L129

Kippenhahn, R., \& Möllenhoff, C. 1974, Ap. Space. Sci., 31, 117

Langer, N., Deutschmann, A., Wellstein, S., \& Höflich P. 2000, A\&A, 362,1046

Langer, N., Yoon, S.-C., Petrovic, J., \& Heger, A. 2004, in Stellar Rotation, Proc. IAU-Symp., 215 (San Francisco: ASP), ed. A.

Maeder, P. Eenens, in press [arXiv: astro-ph/0302232]

Leibundgut, B. 2000, A\&ARv, 10, 179

Leibundgut, B. 2001, ARA\&A, 39, 67

Li, X.-D., \& van den Heuvel, E. P. J. 1997, A\&A, 322, L9

Lindblom, L. 1999, Phys. Rev. D, 60, 4007

Lisewski, A. M., Hillebrandt, W., \& Woosley, S. E. 2000, ApJ, 538, 831

MacFadyen, A. I., \& Woosley, S. E. 1999, ApJ, 524, 262

Maeder, A., \& Meynet, G. 2000, ARA\&A, 38, 143

Middleditch, J. 2004, ApJ, 601, L167

Nomoto, K. 1982, ApJ, 253, 798 
Nomoto, K., \& Kondo, Y. 1991, ApJ, 367, 19

Ostriker, J. P., \& Bodenheimer, P. 1968, ApJ, 151, 1089

Ostriker, J. P., \& Mark, J. W.-K. 1968, ApJ, 151, 1075

Ostriker, J. P., \& Tassoul, J.-L. 1969, ApJ, 155, 987

Paczyński, B. 1991, ApJ, 370, 597

Paczyński, B. 1998, ApJ, 494, L45

Petrovic, J., Langer, N., \& van der Hucht, K. 2005a, A\&A, submitted

Petrovic, J., Langer, N., Yoon, S.-C., \& Heger, A. 2005b, A\&A, submitted

Piersanti, L., Gagliardi, S., Iben, I., \& Tornambé, A. 2003a, ApJ, 583, 885

Piersanti, L., Gagliardi, S., Iben, I., \& Tornambé, A. 2003b, ApJ, 598, 1229

Popham, R., \& Narayan, P. 1991, ApJ, 370, 604

Press, W. H., Flannery, B. P., Teukolsky, S. A., \& Vetterling, W. T. 1986, Numerical Recipes (Cambridge Univ. Press)

Röpke, F. K., \& Hillebrandt, W. 2004, A\&A, 420, L1

Ruderman, M. A., Tao, L., \& Kluźniak, W. 2000, ApJ, 542, 243

Saio, H., \& Nomoto, K. 1998, ApJ, 500, 388

Saio, H., \& Nomoto, K. 2004, ApJ, 615, 444
Tassoul, J.-L. 1978, Theory of Rotating Stars (Princeton: Princeton Univ. Press)

Timmes, F. X., Brown, E. F., \& Truran, J. W. 2003, ApJ, 590, L83

Uenishi, T., Nomoto, K., \& Hachisu, I. 2003, ApJ, 595, 1094

Umeda, H., Nomoto, K., Kobayashi, C., Hachisu, I., \& Kato, M. 1999, ApJ, 522, L43

Usov, V. V. 1992, Nature, 357, 472

Wang, L., Baade, D., Höflich, P., et al. 2003, ApJ, 591, 1110

Wheeler, J. C., Harkness, R. P., Khokhlov, A. M., \& Höflich, P. 1995, Phys. Rep., 256, 211

Wheeler, J. C., Yi, I., Höflich, P., \& Wang, L. 2000, ApJ, 537, 810

Woosley, S. E. 1993, ApJ, 405, 273

Woosley, S. E., \& Weaver, T. A. 1994, ApJ, 423, 371

Yi, I., \& Blackman, E. G. 1998, ApJ, 494, L163

Yoon, S.-C., \& Langer, N. 2003, A\&A, 412, L53

Yoon, S.-C., \& Langer, N. 2004a, A\&A, 419, 623 (Paper I)

Yoon, S.-C., \& Langer, N. 2004b, A\&A, 419, 645

Yoon, S.-C., Langer, N., \& Scheithauer, S. 2004a, A\&A, 425, 217

Yoon, S.-C., Langer, N., \& van der Sluys, M. 2004b, A\&A, 425, 207

Zhang, B., \& Mészáros, P. 2001, ApJ, 552, L35 